\title{
ON THE REGULARITY OF THE BLOW-UP SET FOR SEMILINEAR HEAT EQUATIONS
}

\author{
Hatem ZAAG ${ }^{1}$ \\ Courant Institute, NYU, 251 Mercer Street, NY 10012, New York, USA \\ Received 8 October 2000, revised 22 June 2001
}

ABSTRACT. - We consider $u(x, t)$ a blow-up solution of $u_{t}=\Delta u+|u|^{p-1} u$ where $u: \mathbb{R}^{N} \times$ $[0, T) \rightarrow \mathbb{R}, p>1,(N-2) p<N+2$ and either $u(0) \geqslant 0$ or $(3 N-4) p<3 N+8$. The blow-up set $S \subset \mathbb{R}^{N}$ of $u$ is the set of all blow-up points. Under a nondegeneracy condition, we show that if $S$ is continuous, then it is a $C^{1}$ manifold.

(C) 2002 L'Association Publications de l'Institut Henri Poincaré. Published by Elsevier B.V. All rights reserved

RÉSUMÉ. - On considère $u(x, t)$ une solution singulière de $u_{t}=\Delta u+|u|^{p-1} u$ où $u: \mathbb{R}^{N} \times$ $[0, T) \rightarrow \mathbb{R}, p>1,(N-2) p<N+2$ et soit $u(0) \geqslant 0$, soit $(3 N-4) p<3 N+8$. On définit l'ensemble singulier $S \subset \mathbb{R}^{N}$ de $u$ comme étant l'ensemble de tous les points d'explosion. Sous une certaine condition de non dégénérescence, on montre que si $S$ est continu, alors c'est une variété de classe $C^{1}$.

(C) 2002 L'Association Publications de l'Institut Henri Poincaré. Published by Elsevier B.V. All rights reserved

\section{Introduction}

We are concerned in this paper with blow-up phenomena arising in the following semilinear problem:

$$
\begin{aligned}
u_{t} & =\Delta u+|u|^{p-1} u, \\
u(., 0) & =u_{0} \in L^{\infty}\left(\mathbb{R}^{N}\right),
\end{aligned}
$$

where $u(t): x \in \mathbb{R}^{N} \rightarrow u(x, t) \in \mathbb{R}$ and $\Delta$ stands for the Laplacian in $\mathbb{R}^{N}$. We assume in addition the exponent $p>1$ subcritical: if $N \geqslant 3$ then $1<p<(N+2) /(N-2)$. Moreover, we assume that

$$
u_{0} \geqslant 0 \quad \text { or } \quad(3 N-4) p<3 N+8 .
$$

E-mail address: zaag@cims.nyu.edu (H. Zaag).

${ }^{1}$ Current address: École Normale Supérieure, Département de mathématiques et applications, 45 rue d’Ulm, 75005 Paris, France. E-mail: Hatem.Zaag@ens.fr 
This problem has attracted a lot of attention because it captures features common to a whole range of blow-up problems arising in various physical situations, particularly the role of scaling and self-similarity. Without pretending to be exhaustive, we would like nonetheless to mention some related equations: the motion by mean curvature (Soner and Souganidis [23]), vortex dynamics in superconductors (Chapman, Hunton and Ockendon [6], Merle and Zaag [18]), surface diffusion (Bernoff, Bertozzi and Witelski [2]) and chemotaxis (Brenner et al. [4], Betterton and Brenner [3]). However, Eq. (1) is simple enough to be tractable in rigorous mathematical terms, unlike other physical equations.

A solution $u(t)$ to (1) blows-up in finite time if its maximal existence time $T$ is finite. In this case,

$$
\lim _{t \rightarrow T}\|u(t)\|_{H^{1}\left(\mathbb{R}^{N}\right)}=\lim _{t \rightarrow T}\|u(t)\|_{L^{\infty}\left(\mathbb{R}^{N}\right)}=+\infty .
$$

Let us consider such a solution. $T$ is called the blow-up time of $u$. A point $a \in \mathbb{R}^{N}$ is called a blow-up point if

$$
|u(x, t)| \rightarrow+\infty \quad \text { as }(x, t) \rightarrow(a, T)
$$

(this definition is equivalent to the usual local unboundedness definition, thanks to Corollary 2 in [21]). $S$ denotes the blow-up set, that is the set of all blow-up points. From [21], we know that there exists a blow-up profile $u^{*} \in C_{\text {loc }}^{2}\left(\mathbb{R}^{N} \backslash S\right)$ such that

$$
u(x, t) \rightarrow u^{*}(x) \quad \text { in } C_{\mathrm{loc}}^{2}\left(\mathbb{R}^{N} \backslash S\right) \text { as } t \rightarrow T .
$$

The blow-up problem has been addressed in different ways in the literature. A major direction was developed by authors looking for sufficient blow-up conditions on initial data or on the nonlinear term (see Fujita [12], Ball [1], Levine [16] and the review paper by Deng and Levine [7]). The second main direction is about the description of the asymptotic blow-up behavior, locally near a given blow-up point $\hat{a}$ (see Giga and Kohn [13], Bricmont and Kupiainen [5], Herrero and Velázquez [14,24], Merle and Zaag [21]). Given $a \in \mathbb{R}^{N}$ a blow-up point of $u$, we know that up to some scalings, $u$ approaches a particular explicit function near the singularity $(a, T)$ (see [24]). Up to replacing $u$ by $-u$, one of the following two cases occurs:

Case 1: For all $K_{0}>0$,

$$
\sup _{|z| \leqslant K_{0}}\left|(T-t)^{\frac{1}{p-1}} u\left(a+\tilde{Q}_{a} z \sqrt{(T-t)|\log (T-t)|}, t\right)-f_{l_{a}}(z)\right| \rightarrow 0
$$

as $t \rightarrow T$, where $\tilde{Q}_{a}$ is an orthonormal $N \times N$ matrix, $l_{a}=1, \ldots, N$ and

$$
f_{l_{a}}(z)=\left(p-1+\frac{(p-1)^{2}}{4 p} \sum_{i=1}^{l_{a}} z_{i}^{2}\right)^{-\frac{1}{p-1}} .
$$


Case 2: For all $K_{0}>0$,

$$
\sup _{|z| \leqslant K_{0}}\left|(T-t)^{\frac{1}{p-1}} u\left(a+z(T-t)^{\frac{1}{2 k}}, t\right)-\left(p-1+\sum_{|\alpha|=2 k} C_{\alpha} z^{\alpha}\right)^{-\frac{1}{p-1}}\right|
$$

goes to 0 as $t \rightarrow T$, where $k=2,3,4, \ldots, x^{\alpha}=x_{1}^{\alpha_{1}} \cdots x_{N}^{\alpha_{N}}$ and $|\alpha|=\alpha_{1}+\cdots+\alpha_{N}$ if $\alpha=\left(\alpha_{1}, \ldots, \alpha_{N}\right)$ and $\sum_{|\alpha|=2 k} C_{\alpha} x^{\alpha} \geqslant 0$ for all $x \neq 0$.

Remark. - Even though the proof of [24] is given in the positive case, it extends to unsigned solutions under (2).

The description of the blow-up set $S$ is a major issue. Examples where $S$ is a set of isolated points or a sphere are known to exist (see [17] and [19] for isolated points and [13] for the sphere). If these solutions are artificially considered as defined on $\mathbb{R}^{N^{\prime}} \times[0, T)$ where $N^{\prime}>N$, we obtain examples where $S$ consists in a collection of $\left(N^{\prime}-N\right)$-dimensional subspaces or spheres. No other geometric configurations are known to occur. In [26], Velázquez proves the following result:

The $(N-1)$-dimensional Hausdorff measure of $S$ is bounded on compact sets.

No other regularity result is known.

Our first goal in this paper is to improve this result and obtain partial regularity results on $S$ under some reasonable conditions. Let us consider $\hat{a} \in S$. According to [24] (remark after Theorem 2), if (4) occurs with $l=N$ or (6) occurs with $\sum C_{\alpha} x^{\alpha}>0$ for all $x \neq 0$ (no degenerate directions in the function), then the blow-up point is isolated. The question remains open in the other cases. Even if one assumes that $\hat{a}$ is not isolated, it is unclear whether there is a continuum of blow-up points near $\hat{a}$ or not. This question seems to be very difficult. Whatever the answer is, we don't know how $S$ looks like near $\hat{a}$, and how the profile $u^{*}$ is near $S$ (no relevant information on $u^{*}$ near a nonisolated blow-up point was known before). To make our presentation clearer, we restrict to the case $N=2$ and consider $\hat{a}$ a nonisolated point of $S$ such that $\hat{a}$ belongs to a continuous line of blow-up points without being an endpoint. More precisely, we assume that $\hat{a}=a(0) \in \operatorname{Im} a \subset S$ where $a \in C\left((-1,1), \mathbb{R}^{2}\right)$ and for some $\alpha_{0}$,

$$
\begin{aligned}
& \forall \varepsilon>0, a(-\varepsilon, \varepsilon) \text { intersects the complimentary of any } \\
& \text { connected closed cone with vertex at } \hat{a} \text { and angle } \alpha \in\left(0, \alpha_{0}\right]
\end{aligned}
$$

(this is in a way to insure that $\hat{a}$ is not an endpoint).

Assuming that $u$ behaves according to (4) near the singularity $(\hat{a}, T)$, we have the following result:

THEOREM 1 (Regularity of the blow-up set at a point with the behavior (4) assuming $S$ contains a continuum). - Assume $N=2$ and consider $u$ a solution of (1) that blowsup at time $T$ on a set $S$. Consider $\hat{a}=a(0) \in \operatorname{Im} a \subset S$ where $a \in C\left((-1,1), \mathbb{R}^{2}\right)$ and $\hat{a}$ is not an endpoint (in the sense (7)). If $u$ behaves near $(\hat{a}, T)$ as stated in (4), then there are $\delta>0, \delta_{1}>0$ and $\varphi \in C^{1}\left(\left[-\delta_{1}, \delta_{1}\right], \mathbb{R}\right)$ such that

$$
S \cap B(\hat{a}, 2 \delta)=\operatorname{graph} \varphi \cap B(\hat{a}, 2 \delta)=\operatorname{Im} a \cap B(\hat{a}, 2 \delta) .
$$


In particular, $S$ is a $C^{1}$ manifold near the point $\hat{a}$.

We actually have the following refined $C^{1}$ estimate for $\varphi$.

Proposition 2 (Refined $C^{1}$ estimate for $S$ ). - There exists $C_{0}>0$ and $h_{0}$ such that for all $|\xi|<\delta_{1}$ and $|h|<h_{0}$ such that $|\xi+h|<\delta_{1}$, we have:

$$
\left|\varphi(\xi+h)-\varphi(\xi)-h \varphi^{\prime}(\xi)\right| \leqslant C_{0}|h| \sqrt{\frac{\log |\log | h||}{|\log | h||}} .
$$

Remark. - Using the techniques of Fermanian and Zaag [9], we show in [27] that $\varphi$ is actually $C^{1, \alpha}$ for any $\alpha \in\left(0, \frac{1}{2}\right)$.

Remark. - From [24], we know that the limit function at $(\hat{a}, T)$ stated in (4) has a degenerate direction, and that we cannot have two curves of blow-up points intersecting transversally at $\hat{a}$. With our contribution, we eliminate the possibility of two curves meeting tangentially at $\hat{a}$. In particular, there is no cusp at $\hat{a}$, and there is no sequence of isolated blow-up points converging to $\hat{a} \in S$.

Remark. - The case we are considering does exist indeed. The techniques of [19] hold for the one dimensional equation

$$
\partial_{t} v=\partial_{r r}^{2} v+\frac{N-1}{r} \partial_{r} v+|v|^{p-1} v
$$

which is the radial case of (1). Thus, for all $r_{0}>0$, there is a radial solution $u(x, t)=$ $v(|x|, t)$ of (1) such that for all $K_{0}>0$,

$$
\begin{aligned}
& \sup _{|z| \leqslant K_{0}}\left|(T-t)^{\frac{1}{p-1}} v\left(r_{0}+z \sqrt{(T-t)|\log (T-t)|}\right)-f(z)\right| \rightarrow 0 \quad \text { as } t \rightarrow T, \\
& \text { where for all } z \in \mathbb{R}, f(z)=\left(p-1+\frac{(p-1)^{2}}{4 p} z^{2}\right)^{-\frac{1}{p-1}} .
\end{aligned}
$$

The blow-up set of $u$ is the sphere $r_{0} \mathbb{S}^{N-1}$, and near each blow-up point, (4) holds with the degenerate profile $f_{1}$.

The description of the blow-up profile $u^{*}$ defined in (3) near the singularity $(\hat{a}, T)$ is our second concern in this paper. We claim the following:

THEOREM 3 (Blow-up behavior and profile near a blow-up point where $u$ behaves as in (4) assuming $S$ contains a continuum). - With the notations of Theorem 1, there exists $t_{0}<T$ such that for all $K_{0}>0, t \in\left[t_{0}, T\right)$ and $x \in B(\hat{a}, \delta)$ s.t. $d(x, S) \leqslant$ $K_{0} \sqrt{(T-t)|\log (T-t)|}$, we have

$$
\left|(T-t)^{\frac{1}{p-1}} u(x, t)-f\left(\frac{d(x, S)}{\sqrt{(T-t)|\log (T-t)|}}\right)\right| \leqslant C_{0}^{\prime}\left(K_{0}\right) \frac{\log |\log (T-t)|}{|\log (T-t)|},
$$

where $f$ is defined in (9). Moreover, $\forall x \in \mathbb{R}^{N} \backslash S, u(x, t) \rightarrow u^{*}(x)$ as $t \rightarrow T$ with

$$
u^{*}(x) \sim U(d(x, S)) \quad \text { as } d(x, S) \rightarrow 0 \text { and } x \in B(\hat{a}, \delta),
$$


where

$$
U(z)=\left(\frac{8 p}{(p-1)^{2}} \frac{|\log z|}{z^{2}}\right)^{\frac{1}{p-1}} \text { for } z>0 .
$$

Remark. - This is the first time where the blow-up profile $u^{*}$ is derived near a nonisolated point. Indeed, in the earlier work of Velázquez, the behavior along the "tangential" direction of $S$ was not derived. (10) shows that in a tubular neighborhood of $S$, the main term in the blow-up asymptotics is the 1D blow-up profile $f$, function of only the normal coordinate $\pm d(x, S)$.

Remark. - When $p>3$, we show in [27] that up to a nonsingular function, $u$ is a superposition of $1 \mathrm{D}$ blow-up solutions of (1), organized along the normal directions to the blow-up set.

Theorems 1 and 3 hold in higher dimensions $N \geqslant 3$. However, the hypotheses should be stated more carefully. We claim the following:

THEOREM 4 (Regularity of the blow-up set near a point with the behavior (4) assuming $S$ contains a $(N-l)$-dimensional continuum). - Take $N \geqslant 2$ and $l \in\{1$, $\ldots, N-1\}$. Consider $u$ a solution of (1) that blows-up at time $T$ on a set $S$ and take $\hat{a} \in S$ where $u$ behaves locally as stated in (4). Consider $a \in C\left((-1,1)^{N-l}, \mathbb{R}^{N}\right)$ such that $\hat{a}=a(0) \in \operatorname{Im} a \subset S$ and $\operatorname{Im} a$ is at least $(N-l)$-dimensional (in the sense (82)). If $\hat{a}$ is not an endpoint (in the sense (83) given below), then there are $\delta>0, \delta_{1}>0$ and $\varphi \in C^{1}\left(\left[-\delta_{1}, \delta_{1}\right]^{N-l}, \mathbb{R}^{l}\right)$ such that (8) holds and $S$ is a $C^{1}$ manifold near $\hat{a}$. Proposition 2 and Theorem 3 hold as well.

Remark. - If $l=N-1$, then the fact that $\hat{a}$ is not isolated implies that $\operatorname{Im} a$ is at least 1-dimensional near $\hat{a}$.

Remark. - Theorem 4 can be stated without the hypotheses (82) and (83) if we strengthen the assumption on $\operatorname{Im} a$. Indeed, if we already know that $\operatorname{Im} a$ is a $(N-l)$ dimensional differentiable manifold, then we learn from Theorem 4 that $S \backslash \operatorname{Im} a$ is empty, locally near $\hat{a}$, and we get the blow-up profile near $\hat{a}$ as stated in Theorem 3 .

Up to some complications in the notation, the proof of Theorem 4 remains the same as in the case $N=2$. We will show in Section 6 how to adapt the proof of the case $N=2$ to the general case.

The paper is organized as follows. In Section 2, we recall from previous work the self-similar variables technique and a Liouville theorem for Eq. (1). In Section 3, we show the stability of the behavior (4) (with $l=1<2=N$ ) with respect to the blowup point in $\operatorname{Im} a$. The regularity of the blow-up set is presented in Section 4 where we prove Theorem 1 and Proposition 2. Section 5 is devoted to the blow-up profile of $u$ (Theorem 3). In Section 6, we sketch the proof of Theorem 4. 


\section{Asymptotic behavior in self-similar variables and global estimates for blow-up solutions of (1)}

In this section, we introduce the general framework for the study of $u$ near a singularity $(a, T)$ and recall from [21] a uniform (in space and time) comparison property of $u$ with the solution of the associated $\operatorname{ODE} u^{\prime}=u^{p}$.

\subsection{Self-similar variables}

Given $a$ a blow-up point of $u$, we study the behavior of $u$ near the singularity $(a, T)$ through the introduction of the function $w_{a}$ defined by

$$
w_{a}(y, s)=(T-t)^{\frac{1}{p-1}} u(x, t), \quad y=\frac{x-a}{\sqrt{T-t}}, s=-\log (T-t) .
$$

From (1), we see that $w_{a}$ satisfies for all $(y, s) \in \mathbb{R}^{N} \times[-\log T, \infty)$ the following equation

$$
\frac{\partial w}{\partial s}=\Delta w-\frac{1}{2} y \cdot \nabla w-\frac{w}{p-1}+|w|^{p-1} w .
$$

We know from [13] that

$$
\left\|w_{a}\right\|_{L^{\infty}\left(\mathbb{R}^{N} \times[-\log T, \infty)\right)} \leqslant M<\infty
$$

((12) shows that $M$ is independent of $a$ ) and that

$$
w_{a}(y, s) \rightarrow \pm \kappa \equiv \pm(p-1)^{-\frac{1}{p-1}} \quad \text { as } s \rightarrow \infty
$$

in $L_{\rho}^{2}$ where $\rho(y)=e^{-|y|^{2} / 4} /(4 \pi)^{N / 2}$ and uniformly on compact sets. Assuming that $w_{a} \rightarrow \kappa$, we define

$$
v_{a}=w_{a}-\kappa
$$

We know from (15) and (13) that $\left\|v_{a}\right\|_{L_{\rho}^{2}} \rightarrow 0$ as $s \rightarrow \infty$ and for all $(y, s) \in \mathbb{R}^{N} \times$ $[-\log T, \infty)$,

$$
\frac{\partial v_{a}}{\partial s}=\mathcal{L} v_{a}+f\left(v_{a}\right) \equiv \mathcal{L} v_{a}+\frac{p}{2 \kappa} v_{a}^{2}+g\left(v_{a}\right)
$$

where $\mathcal{L}=\Delta-\frac{1}{2} y . \nabla+1,\left|f\left(v_{a}\right)\right| \leqslant C(M)\left|v_{a}\right|^{2}$ and $\left|g\left(v_{a}\right)\right| \leqslant C(M)\left|v_{a}\right|^{3}$. Operator $\mathcal{L}$ is self-adjoint on $L_{\rho}^{2}$, its spectrum is spec $\mathcal{L}=\left\{1-\frac{m}{2} \mid m \in \mathbb{N}\right\}$. Its eigenfunctions are derived from the Hermite polynomials. If $N=1$, all the eigenvalues of $\mathcal{L}$ are simple. To $1-\frac{m}{2}$ corresponds the eigenfunction

$$
h_{m}(y)=\sum_{n=0}^{\left[\frac{m}{2}\right]} \frac{m !}{n !(m-2 n) !}(-1)^{n} y^{m-2 n} .
$$


If $N \geqslant 2$, then the eigenfunctions corresponding to $1-\frac{m}{2}$ are

$$
H_{\alpha}(y)=h_{\alpha_{1}}\left(y_{1}\right) \cdots h_{\alpha_{N}}\left(y_{N}\right), \quad \text { with } \alpha=\left(\alpha_{1}, \ldots, \alpha_{N}\right) \text { and }|\alpha|=m .
$$

In particular:

- 1 is an eigenvalue of multiplicity 1 and its eigenfunction is $H_{0}(y)=1$,

- $\frac{1}{2}$ is of multiplicity $N$ and its eigenspace is generated by the orthogonal basis $\left\{y_{i} \mid i=1, \ldots, N\right\}$,

- 0 is of multiplicity $\frac{N(N+1)}{2}$ and its eigenspace is generated by the orthogonal basis

$$
\left\{y_{i} y_{j} \mid i<j\right\} \cup\left\{y_{i}^{2}-2 \mid i=1, \ldots, N\right\} .
$$

Since the eigenfunctions of $\mathcal{L}$ make a total orthonormal family of $L_{\rho}^{2}$, we expand $v_{a}$ as follows

$$
v_{a}(y, s)=\sum_{m=0}^{2} v_{a, m}(y, s)+v_{a,-}(y, s) \equiv v_{a, 2}(y, s)+v_{a,-}(y, s)+v_{a,+}(y, s),
$$

where $v_{a, m}(y, s)$ is the orthogonal projection of $v_{a}$ on the eigenspace of $\lambda=1-\frac{m}{2}$, $v_{a,-}(y, s)=P_{-}\left(v_{a}\right)(y, s)$ and $P_{-}$is the projector on the negative subspace of $\mathcal{L}$. Let us define a $N \times N$ symmetric matrix $A_{a}(s)$ by

$$
A_{a}(s)=\int_{\mathbb{R}^{N}} v_{a}(y, s) M(y) \rho(y) \mathrm{d} y \quad \text { where } M_{i, j}(y)=\frac{1}{4} y_{i} y_{j}-\frac{1}{2} \delta_{i j} .
$$

Then, from (19), (18) and the orthogonality between eigenfunctions of $\mathcal{L}$, we have

$$
v_{a, 2}(y, s)=\frac{1}{2} y^{\mathrm{T}} A_{a}(s) y-\operatorname{tr} A_{a}(s) .
$$

From Filippas and Liu [11] and Velázquez [25], we know that

$$
\text { either } v_{a} \sim v_{a, 2} \quad \text { or } \quad v_{a} \sim v_{a,-} \quad \text { in } L_{\rho}^{2} \text { as } s \rightarrow \infty .
$$

In the former case, we know that for some $l_{a} \in\{1, \ldots, N\}, \delta_{a}>0$ and a $N \times N$ orthogonal matrix $\tilde{Q}_{a}$, we have

$$
v_{a}\left(\tilde{Q}_{a} y, s\right)=\frac{\kappa}{2 p s}\left(l_{a}-\frac{1}{2} \sum_{i=1}^{l_{a}} y_{i}^{2}\right)+\mathrm{O}\left(\frac{1}{s^{1+\delta_{a}}}\right) \quad \text { as } s \rightarrow \infty
$$

in $L_{\rho}^{2}$ and $u$ behaves near $(a, T)$ as stated in (4).

If $l_{a}=N$, then $a$ is an isolated blow-up point. We proved in [8] with Fermanian and Merle the stability of such a behavior with respect to perturbations in initial data.

In this paper, we consider the case $l_{a}<N$ and assume that $a$ is not isolated. Although the techniques of [8] imply that this profile is unstable with respect to perturbations in initial data, we will show in Section 3 its stability with respect to the blow-up point (for a fixed solution), in the smaller class of non-isolated blow-up points. 


\subsection{A Liouville theorem and ODE comparison for $u$}

The following rigidity theorem (from [21]) is crucial in the blow-up study of (1). It is a central argument in the proof of our theorem.

Proposition 2.1 (A Liouville theorem for Eq. (1)). - Let $u$ be a solution of (1) defined for all $(x, t) \in \mathbb{R}^{N} \times(-\infty, T)$ such that for some $C>0$,

$$
|u(x, t)| \leqslant \frac{C}{(T-t)^{\frac{1}{p-1}}} .
$$

Then, either $u \equiv 0$ or there exist $T_{1} \in[T,+\infty)$ and $\omega_{0} \in\{-1,+1\}$ such that

$$
u(x, t)=\omega_{0} \kappa\left(T_{1}-t\right)^{-\frac{1}{p-1}} .
$$

This allows Merle and Zaag [21] to prove for $u_{0} \in C^{2}$ the following localization property which reduces the study of the evolution of $u(b, t)$ for a fixed $b$ to the study of an ODE:

PROPOSITION 2.2 (Uniform ODE comparison of blow-up solutions of (1)). - For all $\varepsilon>0$, there exists $C=C\left(\varepsilon,\left\|u_{0}\right\|_{C^{2}}, T\right)$ such that $\forall(x, t) \in \mathbb{R}^{N} \times[0, T)$,

$$
\left.\left.\left|\partial_{t} u-\right| u\right|^{p-1} u|\leqslant \varepsilon| u\right|^{p}+C .
$$

As a consequence, we have the following criterion for regular points (by definition, nonblow-up points):

PROPOSITION 2.3 (Blow-up exclusion criterion). - For all $\varepsilon_{0}>0$, there exists $t_{0}(\varepsilon)<T$ such that if $|u(a, t)| \leqslant\left(1-\varepsilon_{0}\right) \kappa(T-t)^{-1 /(p-1)} \equiv\left(1-\varepsilon_{0}\right) v_{T}(t)$ for some $a \in \mathbb{R}^{N}$ and $t \in\left[t_{0}\left(\varepsilon_{0}\right), T\right)$, then $a$ is not a blow-up point.

Remark. $-v_{T}$ is the solution of $v_{T}^{\prime}=v_{T}^{p}, v_{T}(T)=\infty$.

Proof. - See Corollary 1 in [20] where the criterion is derived from the ODE comparison (note that in [20] the criterion holds only for positive data, but since we show in [21] the ODE comparison for unsigned data, the criterion holds in this general case).

\section{Stability of the blow-up behavior (4) with respect to nonisolated blow-up points}

From now on, we take $1=l<N=2$. We consider $\hat{a}$ a blow-up point of $u$ such that $\hat{a}=a(0)$ where $a \in C\left((-1,1), \mathbb{R}^{2}\right)$ and $\hat{a}$ is not an endpoint of $\operatorname{Im} a \subset S$ in the sense (7). We assume that $u$ has the behavior (4) near $(\hat{a}, T)$. From rotation and translation invariance, we assume that $\hat{a}=0$ and $\tilde{Q}_{\hat{a}}=$ Id. Thus, (4) implies that

$$
\sup _{|z| \leqslant K_{0}}\left|(T-t)^{\frac{1}{p-1}} u(z \sqrt{(T-t)|\log (T-t)|}, t)-f\left(z_{1}\right)\right| \rightarrow 0 \quad \text { as } t \rightarrow T,
$$


where $f$ is defined in (9). Since $u$ has the behavior (24) near $(0, T)$, we know from the previous section (see (22) and (23)) that

$$
v_{0} \sim v_{0,2} \quad \text { and } \quad w_{0}(y, s)-\kappa=v_{0}(y, s) \sim \frac{\kappa}{2 p s}\left(1-\frac{y_{1}^{2}}{2}\right) \quad \text { as } s \rightarrow \infty
$$

in $L_{\rho}^{2}$, where $v_{0}$ and $w_{0}$ are defined in (12) and (16). In the following, we will write $a$ instead of $a(\sigma)$ and $v_{a}$ instead of $v_{a(\sigma)}$. A central argument in our proof is the following:

PROPOSITION 3.1 (Stability of the $L_{\rho}^{2}$ asymptotic behavior with respect to blow-up points in $\operatorname{Im} a)$. - There exist $\sigma_{0}>0, C_{0}>0$ and $s_{0} \in \mathbb{R}$ such that for all $b \in a\left(-\sigma_{0}, \sigma_{0}\right)$, there exists $Q_{b} a 2 \times 2$ orthogonal matrix such that:

(i) for all $|\sigma|<\sigma_{0}$ and $s \geqslant s_{0}$,

$$
\left\|w_{a}\left(Q_{a} y, s\right)-\left\{\kappa+\frac{\kappa}{2 p s}\left(1-\frac{y_{1}^{2}}{2}\right)\right\}\right\|_{L_{\rho}^{2}} \leqslant C_{0} \frac{\log s}{s^{2}} .
$$

(ii) $Q_{0}=\operatorname{Id}$ and $b \in a\left(-\sigma_{0}, \sigma_{0}\right) \rightarrow Q_{b}$ is continuous.

(iii) For all $K_{0}>0$, there is $C_{0}^{\prime}\left(K_{0}\right)>0$ such that for all $s \geqslant s_{0}$,

$$
\sup _{|\sigma|<\sigma_{0},|y| \leqslant K_{0} \sqrt{s}}\left|w_{a}\left(Q_{a} y, s\right)-f\left(\frac{y_{1}}{\sqrt{s}}\right)\right| \leqslant C_{0}^{\prime}\left(K_{0}\right) \frac{\log s}{s}
$$

where $f$ is defined in (9).

Remark. - This argument is similar to the result of [8], where we proved the stability of the blow-up behavior (4) with $l=N$ (the isolated blow-up point case), with respect to initial data. Therefore, we will refer to [8] for the similar steps.

The proof of this proposition follows from 4 steps.

- In Step 1, we show that the control of $v_{a}$ near the same asymptotic $L_{\rho}^{2}$ behavior as $v_{0}$ reduces to the control of its neutral mode $v_{a, 2}$, that is the matrix $A_{a}$ defined in (20) and (21) (this is a finite-dimensional problem).

- In Step 2, we show that the eigenvalues of $A_{a}(s)$ have uniformly the same behavior as those of $A_{0}(s)$ as $s \rightarrow+\infty$.

- In Step 3, we solve the finite-dimensional problem by finding the long time behavior of $A_{a}$.

- In Step 4, we give the solution of the infinite-dimensional problem (that is the asymptotics of $w_{a}$ as $s \rightarrow \infty$ ), which concludes the proof of Proposition 3.1.

\section{Step 1: Uniform reduction to a finite-dimensional problem}

In this step, the only relevant information on $v_{0}$ we use is that $v_{0} \sim v_{0,2}$. We aim at showing that this extends to any $a(\sigma)$ near 0 . In particular, the fact that the asymptotic behavior in (25) has a degenerate direction is not relevant here. Thus, this step is not new. It is exactly the same as the analogous one in the proof of the stability of the profile (4) with $l=N$ presented in [8]. Therefore, we just summarize the arguments of the proof in Appendix A. Let us just remark that the Liouville theorem (Proposition 2.1) is the central argument in getting the uniformity. We claim the following: 
Proposition 3.2 (Reduction to a finite-dimensional problem). - There exists $\sigma_{1}>$ 0 such that for all $\varepsilon>0$, there is $s_{1}(\varepsilon)$ such that for all $|\sigma|<\sigma_{1}$,

$$
\forall s \geqslant s_{1}(\varepsilon)\left\{\begin{array}{l}
\left\|v_{a}(s)\right\|_{L_{\rho}^{2}} \leqslant \varepsilon, \quad\left\|v_{a}-v_{a, 2}(s)\right\|_{L_{\rho}^{2}} \leqslant \varepsilon\left\|v_{a, 2}(s)\right\|_{L_{\rho}^{2}}, \\
\left|A_{a}^{\prime}(s)-\frac{1}{\beta} A_{a}(s)^{2}\right| \leqslant \varepsilon\left|A_{a}(s)\right|^{2},
\end{array}\right.
$$

where $\beta=\frac{\kappa}{2 p}$ and $v_{a, 2}$ and $A_{a}$ are defined in (19), (21) and (20).

Proof. - See Appendix A.

\section{Step 2: A spectral study of the finite-dimensional problem}

In Steps 2 and 3, we solve the finite-dimensional problem given by Step 1. Since $A_{a}$ is a symmetric matrix, we can define its eigenvalues as follows:

LEMmA 3.1 (Existence of regular eigenvalues for $A_{a}$ ). - There exist 2 real $C^{1}$ functions $l_{a, i}(s), i=1,2$, eigenvalues of $A_{a}(s)$. Moreover, the set $\left\{l_{a, 1}(s), l_{a, 2}(s)\right\}$ is continuous in terms of $(a, s) \in S \times[-\log T, \infty)$.

Proof. - From the regularity of $w_{a}$, it is clear that for each $a \in \mathbb{R}^{N}$, the symmetric matrix $A_{a}(s)$ is a $C^{1}$ function of $s$. Therefore, according to Kato [15], we can define 2 $C^{1}$ functions of $s, l_{a, 1}(s)$ and $l_{a, 2}(s)$, eigenvalues of $A_{a}(s)$ (see Lemma 3.2 in [11] for a statement). Since $A_{a}(s)$ is a continuous function of $(a, s)$ and the eigenvalues of a matrix vary continuously with respect to the coefficients, $\left\{l_{a, 1}(s), l_{a, 2}(s)\right\}$ is continuous in terms of $(a, s)$.

Proposition 3.2 and Section 2.1 have the following corollary:

COROLlary 3.1.-

(i) (Nonuniform behavior of $v_{a}$ ) For all $|\sigma|<\sigma_{1}$, (23) holds with $l_{a}=1$. In particular,

$$
A_{a}(s)=-\frac{\beta}{s} \tilde{Q}_{a}\left(\begin{array}{ll}
1 & 0 \\
0 & 0
\end{array}\right) \tilde{Q}_{a}^{T}+\mathrm{O}\left(s^{-1-\delta_{a}}\right) \quad \text { as } s \rightarrow \infty,
$$

and one eigenvalue is equal to $-\frac{\beta}{s}+\mathrm{O}\left(s^{-1-\delta_{a}}\right)$ while the other is equal to $\mathrm{O}\left(s^{-1-\delta_{a}}\right)$ as $s \rightarrow \infty$.

(ii) (Equations on eigenvalues) For all $\varepsilon>0$, there is $s_{1}(\varepsilon)$ such that for all $i \in\{1,2\}$, $|\sigma|<\sigma_{1}$ and $s \geqslant s_{1}(\varepsilon)$

$$
\left|l_{a, i}^{\prime}(s)-\frac{1}{\beta} l_{a, i}(s)^{2}\right| \leqslant \varepsilon\left(l_{a, 1}^{2}+l_{a, 2}^{2}\right) .
$$

Proof. - (i) From Proposition 3.2, we have $v_{a} \sim v_{a, 2}$ as $s \rightarrow \infty$ for all $|\sigma|<\sigma_{1}$, hence (23) holds as stated in Section 2.1. Since $\sigma \rightarrow a(\sigma)$ is continuous and $a(0)=0$ is not an isolated blow-up point (otherwise, (7) cannot hold), every $a(\sigma)$ is nonisolated in $S$. Therefore, $1 \leqslant l_{a}<N=2$ in (23), hence $l_{a}=1$. (20) then gives the estimate for $A_{a}$, which gives the estimate for the eigenvalues. 
(ii) Since $\left(l_{a, 1}^{2}+l_{a, 2}^{2}\right)^{1 / 2}$ is a norm for $A_{a}$, just evaluate the equation on $A_{a}$ in Proposition 3.2 at eigenfunctions to get (ii). This concludes the proof of Corollary 3.1.

At the point $a(0)=0$, we have from Corollary 3.1,

$$
\lambda_{0}(s) \sim-\frac{\beta}{s} \quad \text { and } \quad \mu_{0}(s)=\mathrm{o}\left(\frac{1}{s}\right) \quad \text { as } s \rightarrow \infty
$$

where $\lambda_{0}$ and $\mu_{0}$ are just $l_{0,1}$ and $l_{0,2}$ renamed. This behavior is in fact stable with respect to $\sigma$. In the following proposition, we refine the estimates of Proposition 3.2 and state this stability result.

PROPOSITION 3.3 (Stability of the behavior at infinity of the eigenvalues of $A_{a}(s)$ ). There exists $\sigma_{2}>0, s_{2} \in \mathbb{R}$ and $C_{2}>0$ such that for all $|\sigma|<\sigma_{2}$ and $s \geqslant s_{2}$,

(i) $\left\|v_{a}(s)-v_{a, 2}(s)\right\|_{L_{\rho}^{2}} \leqslant C_{2} s^{-2}$,

(ii) $\left|A_{a}^{\prime}(s)-\frac{1}{\beta} A_{a}(s)^{2}\right| \leqslant C_{2} s^{-3}$,

(iii) $\left|\lambda_{a}(s)+\frac{\beta}{s}\right| \leqslant C_{2} s^{-2} \log s$ and $\left|\mu_{a}(s)\right| \leqslant C_{2} s^{-2}$, where $\lambda_{a}=l_{a, \tau_{a}(1)}, \mu_{a}=l_{a, \tau_{a}(2)}$ and $\tau_{a}$ is a permutation of $\{1,2\}$.

Let us first explain our argument for this proposition formally.

Up to the third order term, the eigenvalues satisfy the equation $\lambda^{\prime}=\frac{1}{\beta} \lambda^{2}$, which has two orbits going to zero as $s \rightarrow \infty$ :

$$
\lambda_{1}(s)=-\frac{\beta}{s+s_{0}} \quad \text { and } \quad \lambda_{2}(s) \equiv 0 .
$$

It is clear that $\lambda_{1}$ is stable, whereas $\lambda_{2}$ is not. Therefore, the stability of the behavior of $\lambda_{0}$ in (27) comes from the dynamical stability analysis of $\lambda_{1}$. This argument was enough in [8] where all the eigenvalues were of order $-\frac{\beta}{s}$ (nondegenerate profile). However, the stability analysis of $\lambda_{2}$ suggests that $\mu_{a}$ is not stable and does not allow us to derive the stability of its behavior. We need a new argument. $\lambda_{2}$ turns out to be stable if $s$ is decreasing from $\infty$ to some point. Corollary 3.1 implies that one eigenvalue (the degenerate direction) of $A_{a}(s)$ is o $\left(\frac{1}{s}\right)$ at infinity, say equal to $\lambda_{2}(s)$ at infinity, up to the order $o\left(\frac{1}{s}\right)$. Thus, we recover the stability of the degenerate eigenvalue. We now give the actual proof.

Proof of Proposition 3.3. - The proof is done in several steps. Let us sketch the main lemmas and derive the proposition first. Thus, we let the lemmas' proof to the end.

Let us fix $\hat{\varepsilon}=\min \left(\frac{1}{2}, \frac{1}{100 \beta}\right)$ and $s_{3}=s_{1}(\hat{\varepsilon})$ defined in Proposition 3.2. From (27) and the continuity of the set of eigenvalues with respect to $a$, we can find $\sigma_{3} \in\left(0, \sigma_{1}\right)$ where $\sigma_{1}$ appears in Corollary 3.1, such that for all $|\sigma| \leqslant \sigma_{3}$,

$$
\left|l_{a, \tau_{a}(1)}\left(s_{3}\right)+\frac{\beta}{s_{3}}\right|+\left|l_{a, \tau_{a}(2)}\left(s_{3}\right)\right| \leqslant \frac{\beta}{100 s_{3}},
$$


where $\tau_{a}$ is a permutation of $\{1,2\}$. Let us rename the eigenvalues such that $\lambda_{a}=l_{a, \tau_{a}(1)}$ and $\mu_{a}=l_{a, \tau_{a}(2)}$. Therefore,

$$
\forall|\sigma| \leqslant \sigma_{3}, \quad\left|\lambda_{a}\left(s_{3}\right)+\frac{\beta}{s_{3}}\right| \leqslant \frac{\beta}{100 s_{3}} \quad \text { and } \quad\left|\mu_{a}\left(s_{3}\right)\right| \leqslant \frac{\beta}{100 s_{3}} .
$$

We claim the following:

LEMMA 3.2 (Nondegeneracy of the decay rate of $v_{a}$ ). - There exists $C_{3}>0$ such that for all $|\sigma|<\sigma_{3}$ and $s \geqslant s_{3}$,

(i) $N_{a}(s) \equiv \lambda_{a}^{2}+\mu_{a}^{2} \geqslant \beta^{2} /\left(16 s^{2}\right)$,

(ii) $\left\|v_{a}(s)\right\|_{L_{\rho}^{2}} \geqslant C_{3} / s$.

We then prove the stability for the nondegenerate direction.

LEMMA 3.3 (Stability of the nondegenerate direction of $A_{a}(s)$ ). - For all $|\sigma|<\sigma_{3}$ and $s \geqslant s_{3}$,

$$
-\frac{2 \beta}{s} \leqslant \lambda_{a}(s) \leqslant-\frac{\beta}{2 s} \text { and }-\frac{2 \beta}{s} \leqslant \mu_{a}(s) \leqslant \frac{C}{s} .
$$

With this lemma, we can refine the equation satisfied by $\lambda_{a}$ and $\mu_{a}$.

LEMMA 3.4 (A refined equation satisfied by $A_{a}(s)$ ). - There exists $s_{4} \geqslant s_{3}$ and $C_{4}>0$ such that for all $|\sigma|<\sigma_{3}$ and $s \geqslant s_{4}$,

$$
\begin{aligned}
& \left\|v_{a}-v_{a, 2}\right\|_{L_{\rho}^{2}} \equiv\left(\left\|v_{a,+}(s)\right\|_{L_{\rho}^{2}}^{2}+\left\|v_{a,-}(s)\right\|_{L_{\rho}^{2}}^{2}\right)^{1 / 2} \leqslant C_{4} s^{-2}, \\
& \left|A_{a}^{\prime}(s)-\frac{1}{\beta} A_{a}(s)^{2}\right|+\left|\lambda_{a}^{\prime}-\frac{1}{\beta} \lambda_{a}^{2}\right|+\left|\mu_{a}^{\prime}-\frac{1}{\beta} \mu_{a}^{2}\right| \leqslant C_{4} s^{-3} .
\end{aligned}
$$

Lemma 3.3 and Corollary 3.1 imply that for all $|\sigma|<\sigma_{3}$,

$$
\mu_{a}(s)=\mathrm{O}\left(s^{-1-\delta_{a}}\right) \quad \text { as } s \rightarrow \infty .
$$

Eq. (30) propagates this estimate from $\infty$ to $s$ and improves it. More precisely,

LEMma 3.5 (Stability of the degenerate direction of $A_{a}(s)$ ). - There exist $s_{5} \geqslant s_{4}$ and $C_{5}>0$ such that for all $|\sigma|<\sigma_{3}$ and $s \geqslant s_{5}$,

$$
\left|\mu_{a}(s)\right| \leqslant C_{5} s^{-2},
$$

With this information, we can refine the estimate on $\lambda_{a}(s)$.

LEMMA 3.6 (Refinement of the estimate on the nondegenerate direction of $A_{a}(s)$ ). There exist $s_{6} \geqslant s_{5}, \sigma_{6}<\sigma_{3}$ and $C_{6}>0$ such that for all $|\sigma|<\sigma_{6}$ and $s \geqslant s_{6}$,

$$
\left|\lambda_{a}(s)+\frac{\beta}{s}\right| \leqslant C_{6} \frac{\log s}{s^{2}} .
$$

It is clear that Lemmas 3.4, 3.5 and 3.6 directly imply Proposition 3.3. Let us now prove the previous lemmas. 
Proof of Lemma 3.2. - Recall that $\hat{\varepsilon}, s_{3}$ and $\sigma_{3}$ are defined just before (28).

(i) From Corollary 3.1, we have for all $|\sigma|<\sigma_{3}$ and $s \geqslant s_{3}$,

$$
N_{a}^{\prime}(s)=2\left(\lambda_{a} \lambda_{a}^{\prime}+\mu_{a} \mu_{a}^{\prime}\right) \geqslant \frac{2}{\beta}\left(\lambda_{a}^{3}+\mu_{a}^{3}\right)-2 \hat{\varepsilon}\left(\lambda_{a}+\mu_{a}\right)\left(\lambda_{a}^{2}+\mu_{a}^{2}\right) \geqslant-\frac{6}{\beta} N_{a}^{3 / 2}
$$

(here we used the fact that $\hat{\varepsilon} \leqslant \frac{1}{100 \beta}$ and $\left|\lambda_{a}^{n}+\mu_{a}^{n}\right| \leqslant 2\left(\lambda_{a}^{2}+\mu_{a}^{2}\right)^{n / 2}$ ).

Since $N_{a}\left(s_{3}\right)>\beta^{2} /\left(16 s_{3}^{2}\right)$ (from $\left.(28)\right)$ and

$$
\frac{\mathrm{d}}{\mathrm{d} s}\left(\frac{\beta^{2}}{16 s^{2}}\right)<-\frac{6}{\beta}\left(\frac{\beta^{2}}{16 s^{2}}\right)^{3 / 2}
$$

straightforward a priori estimates yield (i).

(ii) Since $\hat{\varepsilon} \leqslant \frac{1}{2}$, Proposition 3.2 implies that $\left\|v_{a}\right\|_{L_{\rho}^{2}} \geqslant \frac{1}{2}\left\|v_{a, 2}\right\|_{L_{\rho}^{2}} \geqslant C\left(\lambda_{a}^{2}+\mu_{a}^{2}\right)^{1 / 2}$ where $C>0$ (because $\left(\lambda_{a}^{2}+\mu_{a}^{2}\right)^{1 / 2}$ is a norm for $A_{a}$, hence for $v_{a, 2}$ by (21)). Thus, (ii) of Lemma 3.2 follows from (i). This concludes the proof of Lemma 3.2.

Proof of Lemma 3.3. - We claim that for all $|\sigma|<\sigma_{3}$ and $s \geqslant s_{3}$,

$$
\lambda_{a}(s)+\mu_{a}(s)<-\frac{\beta}{50 s}
$$

Indeed, from Corollary 3.1, Lemma 3.2 and the fact that $\hat{\varepsilon} \leqslant \frac{1}{100 \beta}$, we have

$$
\forall|\sigma|<\sigma_{3}, \forall s \geqslant s_{3}, \quad \frac{\mathrm{d}}{\mathrm{d} s}\left(\lambda_{a}+\mu_{a}\right) \geqslant\left(\frac{1}{\beta}-2 \hat{\varepsilon}\right)\left(\lambda_{a}^{2}+\mu_{a}^{2}\right) \geqslant \frac{1}{2 \beta} \frac{\beta^{2}}{16 s^{2}}
$$

Since $\lambda_{a}(s)+\mu_{a}(s) \rightarrow 0$ as $s \rightarrow \infty$ (Corollary 3.1), an integration between $s$ and $\infty$ gives (32).

(32) shows that Lemma 3.3 follows if we prove that for all $|\sigma|<\sigma_{3}$ and $s \geqslant s_{3}$,

$$
-\frac{2 \beta}{s}<\lambda_{a}(s)<-\frac{\beta}{2 s} \text { and } \mu_{a}(s)>-\frac{2 \beta}{s} \text {. }
$$

We proceed by contradiction. From (28), we consider some $|\sigma|<\sigma_{3}$ and $s_{*}>s_{3}$ such that (33) holds for all $s \in\left[s_{3}, s_{*}\right.$ ) with an equality case at $s_{*}$. In the following, we rule out those 3 cases of equality. Let us just mention that (33) and (32) yield

$$
\left|\mu_{a}\left(s_{*}\right)\right| \leqslant \frac{2 \beta}{s_{*}} .
$$

Case 1: $\lambda_{a}\left(s_{*}\right)=-\frac{\beta}{2 s_{*}}$.

On one hand, we have

$$
\lambda_{a}^{\prime}\left(s_{*}\right) \geqslant\left.\frac{\mathrm{d}}{\mathrm{d} s}\left(-\frac{\beta}{2 s}\right)\right|_{s=s_{*}} \geqslant \frac{\beta}{2 s_{*}^{2}} .
$$


On the other hand, Corollary 3.1, (33) and (34) imply that

$$
\begin{aligned}
\lambda_{a}^{\prime}\left(s_{*}\right) & \leqslant \frac{1}{\beta} \lambda_{a}\left(s_{*}\right)^{2}+\hat{\varepsilon}\left(\lambda_{a}\left(s_{*}\right)^{2}+\mu_{a}\left(s_{*}\right)^{2}\right) \\
& \leqslant \frac{1}{\beta}\left(\frac{\beta}{2 s_{*}}\right)^{2}+\hat{\varepsilon}\left(\left(\frac{2 \beta}{s_{*}}\right)^{2}+\left(\frac{2 \beta}{s_{*}}\right)^{2}\right) \leqslant \frac{\beta}{3 s_{*}^{2}}
\end{aligned}
$$

because $\hat{\varepsilon} \leqslant \frac{1}{100 \beta}$. Contradiction.

Case 2 or $3: \lambda_{a}\left(s_{*}\right)=-\frac{2 \beta}{s_{*}}$ or $\mu_{a}\left(s_{*}\right)=-\frac{2 \beta}{s_{*}}$.

Let us handle for instance Case 3 . Case 2 is exactly the same.

On one hand, we have

$$
\mu_{a}^{\prime}\left(s_{*}\right) \leqslant\left.\frac{\mathrm{d}}{\mathrm{d} s}\left(-\frac{2 \beta}{s}\right)\right|_{s=s_{*}} \leqslant \frac{2 \beta}{s_{*}^{2}} .
$$

On the other hand, Corollary 3.1, (33) and (34) imply that

$$
\begin{aligned}
\mu_{a}^{\prime}\left(s_{*}\right) & \geqslant \frac{1}{\beta} \mu_{a}\left(s_{*}\right)^{2}-\hat{\varepsilon}\left(\lambda_{a}\left(s_{*}\right)^{2}+\mu_{a}\left(s_{*}\right)^{2}\right) \\
& \geqslant \frac{1}{\beta}\left(\frac{2 \beta}{s_{*}}\right)^{2}-\hat{\varepsilon}\left(\left(\frac{2 \beta}{s_{*}}\right)^{2}+\left(\frac{2 \beta}{s_{*}}\right)^{2}\right) \geqslant \frac{3 \beta}{s_{*}^{2}}
\end{aligned}
$$

because $\hat{\varepsilon} \leqslant \frac{1}{100 \beta}$. Contradiction.

Thus, (33) holds for all $|\sigma|<\sigma_{3}$ and $s \geqslant s_{3}$. This concludes the proof of Lemma 3.3.

Proof of Lemma 3.4. - We just follow ideas due to Filippas, Kohn and Liu [10,11]. See Appendix B.

Proof of Lemma 3.5. - An iteration argument for $\mu_{a}(s)$ based on (30) and (31) gives the result. Indeed, these estimates yield $\mu_{a}^{\prime}=\beta^{-1} \mu_{a}^{2}+\mathrm{O}\left(s^{-3}\right)=\mathrm{O}\left(s^{-\left(2+2 \delta_{a}\right)}\right)+\mathrm{O}\left(s^{-3}\right)$ as $s \rightarrow \infty$.

If $2 \delta_{a} \geqslant 1$, then $\mu_{a}=\mathrm{O}\left(\frac{1}{s^{2}}\right)$. If $2 \delta_{a}<1$, then $\mu_{a}=\mathrm{O}\left(\frac{1}{s^{1+2 \delta_{a}}}\right)$. In this case, we repeat the same argument with $2 \delta_{a}$ instead of $\delta_{a}$ until we get

$$
\forall|\sigma|<\sigma_{3}, \quad \mu_{a}(s)=\mathrm{O}\left(\frac{1}{s^{2}}\right) \quad \text { as } s \rightarrow \infty .
$$

Fix $s_{5} \geqslant s_{4}$ such that

$$
\forall s \geqslant s_{5}, \quad\left(C_{4}+\frac{1}{\beta}\right) \frac{1}{2 s^{2}}<\frac{1}{s^{7 / 4}},
$$

where $s_{4}$ and $C_{4}$ are defined in Lemma 3.4. From (35), we can define for all $|\sigma|<\sigma_{3}$,

$$
s_{\sigma}^{*}=\min \left\{s^{*} \geqslant s_{5}\left|\forall s \geqslant s^{*},\right| \mu_{a}(s) \mid \leqslant s^{-7 / 4}\right\} .
$$

Using (30), we have for all $s \in\left[s_{\sigma}^{*}, \infty\right)$,

$$
\left|\mu_{a}^{\prime}(s)\right| \leqslant \beta^{-1}\left|\mu_{a}(s)\right|^{2}+C_{4} s^{-3} \leqslant\left(C_{4}+\beta^{-1}\right) s^{-3} .
$$


Therefore,

$$
\forall s \in\left[s_{\sigma}^{*}, \infty\right), \quad\left|\mu_{a}(s)\right| \leqslant\left(C_{4}+\beta^{-1}\right) s^{-2} / 2<s^{-7 / 4}
$$

since $s_{\sigma}^{*} \geqslant s_{5}$ (see (36)). (37) then shows that $s_{\sigma}^{*}=s_{5}$ and (38) yields the result.

Proof of Lemma 3.6. - Let us define

$$
Z_{a}(s)=s^{2}\left(\lambda_{a}(s)+\frac{\beta}{s}\right) .
$$

From (30) and Corollary 3.1, we have for all $|\sigma|<\sigma_{3}$,

$$
Z_{a}(s)=\mathrm{O}\left(s^{1-\delta_{a}}\right) \quad \text { as } s \rightarrow \infty, \quad \forall s \geqslant s_{4},\left|Z_{a}^{\prime}(s)-\frac{Z_{a}^{2}}{\beta s^{2}}\right| \leqslant C_{4} s^{-1} .
$$

As for Lemma 3.5, we improve the estimate on $Z_{a}$ iteratively.

From (40), we write $Z_{a}^{\prime}=\mathrm{O}\left(s^{-2 \delta_{a}}\right)+\mathrm{O}\left(s^{-1}\right)$.

If $2 \delta_{a} \geqslant 1$, then $Z_{a}=\mathrm{O}(\log s)$. If $2 \delta_{a}<1$, then $Z_{a}(s)=\mathrm{O}\left(s^{1-2 \delta_{a}}\right)$. We repeat the same argument with $2 \delta_{a}$ instead of $\delta_{a}$ until we get

$$
\forall|\sigma|<\sigma_{3}, Z_{a}=\mathrm{O}(\log s), \quad \text { hence } \quad \lambda_{a}=-\frac{\beta}{s}+\mathrm{O}\left(\frac{\log s}{s^{2}}\right)
$$

as $s \rightarrow \infty$. We need to prove that this holds uniformly with respect to $\sigma$. Let us consider $s_{7}$ and $C_{7} \geqslant 2 C_{4}$ such that for all $s \geqslant s_{7},\left|Z_{0}(s)\right| \leqslant C_{7} \log s$ and $Z_{a}(s)$ is continuous in terms of $(a, s) \in S \times\left[s_{7}, \infty\right)$ (for this latter fact, remember from Lemma 3.1 the continuity of $\left\{\lambda_{a}(s), \mu_{a}(s)\right\}$ in terms of $\left.(a, s)\right)$. If $s_{7}$ is chosen so that $C_{5} s_{7}^{-2} \leqslant \frac{\beta}{4} s_{7}^{-1}$, then $\lambda_{a}(s)$ and $\mu_{a}(s)$ become apart for $s \geqslant s_{7}$ by Lemmas 3.3 and 3.5. Therefore, both are continuous in terms of $(a, s) \in S \times\left[s_{7}, \infty\right)$.

Define $s_{6} \geqslant s_{7}$ and then $\sigma_{6} \leqslant \sigma_{3}$ such that

$$
\forall s \geqslant s_{6}, \frac{16 C_{7}^{2} \log ^{2} s}{\beta s^{2}} \leqslant \frac{C_{4}}{s} \quad \text { and } \quad \forall|\sigma| \leqslant \sigma_{6},\left|Z_{a}\left(s_{6}\right)\right| \leqslant 2 C_{7} \log s_{6}
$$

We claim that

$$
\text { for all }|\sigma|<\sigma_{6} \text { and } s \geqslant s_{6}, \quad\left|Z_{a}(s)\right| \leqslant 4 C_{7} \log s .
$$

Indeed, if for some $|\sigma|<\sigma_{6}$ and $s \geqslant s_{6}$, we have $\left|Z_{a}(s)\right|>4 C_{7} \log s$, then we can define from (41) $s_{\sigma}^{*}$ such that

$$
\forall s \in\left[s_{6}, s_{\sigma}^{*}\right], \quad\left|Z_{a}(s)\right| \leqslant 4 C_{7} \log s \text { and }\left|Z_{a}\left(s_{\sigma}^{*}\right)\right|=4 C_{7} \log s_{\sigma}^{*} .
$$

Using (40), (41), and the fact that $C_{7} \geqslant 2 C_{4}$, we have

$$
\forall s \in\left[s_{6}, s_{\sigma}^{*}\right], \quad\left|Z_{a}^{\prime}(s)\right| \leqslant \frac{1}{\beta} \frac{Z_{a}^{2}}{s^{2}}+\frac{C_{4}}{s} \leqslant \frac{16 C_{7}^{2}(\log s)^{2}}{\beta s^{2}}+\frac{C_{4}}{s} \leqslant \frac{2 C_{4}}{s} \leqslant \frac{C_{7}}{s} .
$$


Therefore, $\left|Z_{a}\left(s_{\sigma}^{*}\right)\right| \leqslant\left|Z_{a}\left(s_{6}\right)\right|+C_{7}\left(\log s_{\sigma}^{*}-\log s_{6}\right) \leqslant 3 C_{7} \log s_{\sigma}^{*}$ by (41). This contradicts (43). Thus, (42) holds. This closes the proof of Lemma 3.6 by (39). Thus Proposition 3.3 is proved.

\section{Step 3: Solution of the finite-dimensional problem}

Now, we are ready to solve (26). We claim the following:

Proposition 3.4 (Solution of the finite-dimensional problem). - There exists $C_{10}>$ 0 such that for all $b \in a\left(-\sigma_{2}, \sigma_{2}\right)$, there exists $a 2 \times 2$ orthogonal matrix $Q_{b}$ such that:

$$
\text { for all }|\sigma|<\sigma_{2} \text { and } s \geqslant s_{2}, \quad\left|A_{a}(s)+\frac{\beta}{s} L_{a}\right| \leqslant C_{10} \frac{\log s}{s^{2}},
$$

where

$$
L_{a}=Q_{a}\left(\begin{array}{ll}
1 & 0 \\
0 & 0
\end{array}\right) Q_{a}^{T}
$$

Moreover, $Q_{0}=\operatorname{Id}$ and $b \in a\left(-\sigma_{2}, \sigma_{2}\right) \rightarrow Q_{b}$ is continuous.

Proof. - It is easy to check from Proposition 3.3 that for all $|\sigma|<\sigma_{2}$ and $s \geqslant s_{2}$,

$$
\begin{aligned}
& \left|A_{a}^{\prime}(s)-\frac{\operatorname{tr} A_{a}}{\beta} A_{a}(s)+\operatorname{det} A_{a}(s) \operatorname{Id}\right| \leqslant C{ }^{-3}, \\
& \left|A_{a}(s)\right| \leqslant C s^{-1}, \quad\left|\operatorname{tr} A_{a}+\frac{\beta}{s}\right| \leqslant C s^{-2} \log s, \quad\left|\operatorname{det} A_{a}\right| \leqslant C s^{-3} .
\end{aligned}
$$

Therefore, for all $|\sigma|<\sigma_{2}$ and $s \geqslant s_{2}$,

$$
\left|A_{a}^{\prime}+\frac{1}{s} A_{a}(s)\right| \leqslant C \frac{\log s}{s^{3}}, \quad \text { hence } \quad\left|\frac{\mathrm{d}}{\mathrm{d} s}\left(s A_{a}(s)\right)\right| \leqslant C \frac{\log s}{s^{2}} .
$$

This shows that $-\frac{s}{\beta} A_{a}(s)$ has a limit as $\rightarrow \infty$. This limit depends only on $a(\sigma)$ and not on $\sigma$, for $A_{a}(s)$ does the same (see (20)). Therefore, we call this limit $L_{a(\sigma)}$. We define this way a function $b \in a\left(-\sigma_{2}, \sigma_{2}\right) \rightarrow L_{b} . L_{a(\sigma)}$ is a $2 \times 2$ symmetric matrix, such that for all $|\sigma|<\sigma_{2}$ and $s \geqslant s_{2}$,

$$
\left|s A_{a}(s)+\beta L_{a}\right| \leqslant C \int_{s}^{\infty} t^{-2} \log t \mathrm{~d} t \leqslant C s^{-1} \log s .
$$

Since the convergence is uniform "with respect to $a(\sigma)$ " and since for a fixed $s, A_{a}(s)$ is continuous with respect to $a, b \rightarrow L_{b}$ is continuous.

Since $L_{a}$ is symmetric, it has 2 eigenvalues which are the limits as $s \rightarrow \infty$, of $-\frac{s}{\beta} \lambda_{a}$ and $-\frac{s}{\beta} \mu_{a}$, say 1 and 0 , according to Proposition 3.3. Therefore, since $b \rightarrow L_{b}$ is continuous and $L_{b}$ is symmetric with distinct eigenvalues, we can define a $2 \times 2$ orthogonal matrix $Q_{b}$, continuous in terms of $b$, such that (45) and then (44) hold (just define continuous eigenvectors). From (i) of Corollary 3.1, we can even choose $Q_{0}=\tilde{Q}_{0}$, hence, $Q_{0}=$ Id. 


\section{Step 4: Asymptotic behavior of $w_{a}$ in $L_{\rho}^{2}$}

We prove Proposition 3.1 here. We first use the solution of the finite-dimensional problem to find the asymptotic behavior of $w_{a}$ as $s \rightarrow \infty$, in $L_{\rho}^{2}$ or equivalently uniformly on compact sets of $\mathbb{R}^{N}$. We then use techniques from [24] to extend the convergence up to sets of the type $\left\{|y| \leqslant K_{0} \sqrt{s}\right\}$.

Proof of Proposition 3.1. - (i) Take $\sigma_{0}=\sigma_{2}$ and $s_{0}=s_{2}$ where $\sigma_{2}$ and $s_{2}$ are defined in Proposition 3.3. Consider $|\sigma|<\sigma_{0}$ and $s \geqslant s_{0}$. With the change of variable $z=Q_{a} y$ and using (45), we have

$$
\begin{aligned}
& \left\|w_{a}\left(Q_{a} y, s\right)-\left\{\kappa+\frac{\kappa}{2 p s}\left(1-\frac{y_{1}^{2}}{2}\right)\right\}\right\|_{L_{\rho}^{2}} \\
& =\left\|w_{a}(z, s)-\left\{\kappa+\frac{\beta}{s}\left(1-\frac{\left(Q_{a}^{\mathrm{T}} z\right)_{1}^{2}}{2}\right)\right\}\right\|_{L_{\rho}^{2}}\left(\beta=\frac{\kappa}{2 p}\right) \\
& =\left\|v_{a}(z, s)-\left\{-\frac{\beta}{2 s} z^{\mathrm{T}} L_{a} z+\frac{\beta}{s}\right\}\right\|_{L_{\rho}^{2}} \\
& \quad \leqslant\left\|v_{a}(s)-v_{a, 2}(s)\right\|_{L_{\rho}^{2}}+\left\|v_{a, 2}(s)-\left\{-\frac{\beta}{2 s} z^{\mathrm{T}} L_{a} z+\frac{\beta}{s}\right\}\right\|_{L_{\rho}^{2}} \equiv E_{1}+E_{2} .
\end{aligned}
$$

According to Proposition 3.3, we have

$$
E_{1}=\left\|v_{a}(s)-v_{a, 2}(s)\right\|_{L_{\rho}^{2}} \leqslant \frac{C_{2}}{s^{2}} .
$$

Using (21) and (45), we have

$$
E_{2}=\left\|\frac{1}{2} z^{\mathrm{T}} A_{a}(s) z-\operatorname{tr} A_{a}(s)-\left\{-\frac{\beta}{2 s} z^{\mathrm{T}} L_{a} z-\operatorname{tr}\left(-\frac{\beta}{s} L_{a}\right)\right\}\right\|_{L_{\rho}^{2}} .
$$

Therefore, we have from (44)

$$
E_{2} \leqslant C\left|A_{a}(s)+\frac{\beta}{s} L_{a}\right| \leqslant C C_{10} \frac{\log s}{s^{2}} .
$$

Combining (47), (48) and (50) gives (i) of Proposition 3.1.

(ii) See Proposition 3.4.

(iii) The derivation of (iii) from (i) was done by Velázquez in [24] for a fixed blowup point $a$. However, in [24], the convergence speed was not given, because the error estimate in the $L_{\rho}^{2}$ convergence was not that accurate there. We shall summarize in Appendix $\mathrm{C}$ the method of Velázquez, with a special care to the speed of convergence, and of course, to the uniformity with respect to the blow-up point.

\section{Regularity of the blow-up set near a nonisolated point with the behavior (4)}

\subsection{Continuous differentiability of $S$}

We prove Theorem 1 in this subsection. We proceed in 2 steps: 
- In Step 1, we derive from the stability of the blow-up behavior with respect to blowup points in $\operatorname{Im} a$ a kind of weak differentiability of $S$ at points of $\operatorname{Im} a$ (the cone property).

- In Step 2, we define a $C^{1}$ function $A$ whose image is a graph and which is equal to $S$ in a neighborhood of the origin.

\section{Step 1: The cone property for $\operatorname{Im} a$}

Let us introduce the cone property first.

DEFINITION 4.1 (Cone property and the weak tangent). - Consider a set $E \subset \mathbb{R}^{2}$.

(i) $E$ is said to have the cone property at some $a \in E$ if there is $u \in \mathbb{S}^{1}$ such that for all $\varepsilon>0$, there is $\delta(a, \varepsilon)>0$ such that

$$
E \cap B(a, \delta) \subset \Omega_{a, u, \varepsilon} \equiv\{x||(x-a) \cdot u|\geqslant(1-\varepsilon)| x-a \mid\} .
$$

$\mathbb{R} u$ is then called the weak tangent of $E$ at a.

(ii) $E$ is said to have the uniform cone property at some subset $F \subset E$ if for all $\varepsilon>0$ and $a \in F, E$ has the cone property at a with $\delta(a, \varepsilon)=\delta(\varepsilon)$.

Remark. $-\Omega_{a, u, \varepsilon}$ is a cone with vertex $a$. It shrinks to $a+\mathbb{R} u$ as $\varepsilon \rightarrow 0$.

Remark. - If $E$ is a $C^{1}$ curve, then the cone property is equivalent to the differentiability and the weak tangent to the tangent.

Let us explain our argument first. The function $w_{a(\sigma)}$ defined in (12) describes the local behavior of $u$, near $a(\sigma)$. From (iii) of Proposition 3.1, we see that if we travel along the direction $Q_{a(\sigma)} e_{1}$ from 0 to $y=\eta \sqrt{s}$ where $\eta>0$, then we make $w_{a}(y, s)$ drop down from $f(0)=\kappa$ to $f(\eta)<\kappa$. No change occurs if we travel along $Q_{a(\sigma)} e_{2}$ (hence, we call it the degenerate direction). In the $u(x, t)$ variable, this means that when we travel along the nondegenerate direction $Q_{a(\sigma)} e_{1}$, from $a$ to $x=a+\eta e^{-s / 2} \sqrt{s}$, $u(x, t)$ drops down from $v_{T}(t) \equiv \kappa(T-t)^{-1 /(p-1)}$ to $\left(1-\varepsilon_{0}(\eta)\right) v_{T}(t)$. Therefore, if $s$ is large enough, all points along this nondegenerate direction satisfy the blow-up exclusion criterion of Proposition 2.3. Thus, $S$ is located along the degenerate direction $Q_{a(\sigma)} e_{2}$. More precisely, we have the following:

PROPOSITION 4.1 (Uniform cone property for $S$ at points of $\operatorname{Im} a$ ). -

(i) $S$ has the uniform cone property at $\operatorname{Im} a_{|\sigma|<\sigma_{0}}$. The weak tangent at $a(\sigma)$ is $\mathbb{R} Q_{a(\sigma)} e_{2}$ where $e_{2}=(0,1)$.

(ii) $Q_{0}=$ Id and the weak tangent is continuous as a function of $b \in a\left(-\sigma_{0}, \sigma_{0}\right)$.

Remark. - Velázquez's result in [24] implies that $S$ has the cone property at $a(\sigma)$, but with no uniformity with respect of $a$.

Proof of Proposition 4.1. - Note that (ii) follows directly from (i) of Proposition 4.1 and (ii) of Proposition 3.1. Let us prove (i). We need to prove that for all $\varepsilon>0$, there is $\delta(\varepsilon)$ such that for all $|\sigma|<\sigma_{0}$, if

$$
|x-a|<\delta \quad \text { and } \quad\left|(x-a) \cdot Q_{a} e_{2}\right|<(1-\varepsilon)|x-a|,
$$


then $x \notin S$. Consider $\varepsilon$ and let us first introduce $\delta(\varepsilon)$ and then show that it is convenient. Define

$$
\varepsilon_{0}=\frac{1}{2}(\kappa-f(\sqrt{\varepsilon}))>0 \text { and } t_{0}=t_{0}\left(\varepsilon_{0}\right)
$$

as defined in Proposition 2.3. Consider then $s^{*}(\varepsilon)$ such that

$$
\forall s \geqslant s^{*}(\varepsilon), \quad C_{0}^{\prime}(1) \frac{\log s}{s} \leqslant \varepsilon_{0},
$$

where $C_{0}^{\prime}$ is defined in Proposition 3.1. Define

$$
\delta(\varepsilon)=e^{-\tilde{s} / 2} \sqrt{\tilde{s}}, \quad \text { where } \tilde{s}(\varepsilon)=\max \left(s_{0}+1, s^{*}(\varepsilon),-\log \left(T-t_{0}\right)\right),
$$

where $s_{0}$ is introduced in Proposition 3.1. Let us take any $|\sigma|<\sigma_{0}$ and $x$ as in (52) and show that $x$ is not a blow-up point. We will use the blow-up exclusion criterion of Proposition 2.3. Let us introduce $t_{a, x}$ and similarity variables such that

$$
\begin{aligned}
& |x-a|=\sqrt{\left(T-t_{a, x}\right)\left|\log \left(T-t_{a, x}\right)\right|}, \quad s_{a, x}=-\log \left(T-t_{a, x}\right), \\
& y_{a, x}=Q_{a}^{-1}\left(\frac{x-a}{\sqrt{T-t_{a, x}}}\right) .
\end{aligned}
$$

The following lemma allows us to conclude.

LEMMA 4.3. -
(i) $s_{a, x} \geqslant \max \left(s^{*}(\varepsilon),-\log \left(T-t_{0}\right), s_{0}+1\right)$,
(ii) $t_{a, x} \geqslant t_{0}$,
(iii) $\left|y_{a, x}\right|=\sqrt{s_{a, x}}$,
(iv) $\left|y_{a, x, 2}\right| \leqslant(1-\varepsilon)\left|y_{a, x}\right|$,
(v) $\left|y_{a, x, 1}\right| \geqslant \sqrt{\varepsilon s_{a, x}}$,
(vi) $\left|u\left(x, t_{a, x}\right)\right| \leqslant\left(\kappa-\varepsilon_{0}\right) /\left(T-t_{a, x}\right)^{1 /(p-1)}$.

Indeed, according to (ii) and (vi) of Lemma 4.3 and (53), $x$ satisfies the blow-up exclusion criterion of Proposition 2.3 and is therefore not a blow-up point. Remains to prove Lemma 4.3.

Proof of Lemma 4.3. - (i) From (56), (52) and (55), we have $e^{-s_{a, x} / 2} \sqrt{s_{a, x}}=|x-a| \leqslant$ $\delta=e^{-\tilde{s} / 2} \sqrt{\tilde{s}}$. Therefore, $s_{a, x} \geqslant \tilde{s}$. Use (55) again to conclude.

(ii) Since $s_{a, x}=-\log \left(T-t_{a, x}\right)$, use (i) to conclude.

(iii) From (56), we have $\left|y_{a, x}\right|=|x-a| / \sqrt{T-t_{a, x}}=\sqrt{\left|\log \left(T-t_{a, x}\right)\right|}=\sqrt{s_{a, x}}$.

(iv) From (52), we have $\left|(x-a) \cdot Q_{a} e_{2}\right| \leqslant(1-\varepsilon)|x-a|$. The conclusion follows since $Q_{a} y_{a, x}=(x-a) / \sqrt{T-t_{a, x}}$ by (56).

(v) We have $y_{a, x, 1}^{2}=\left|y_{a, x}\right|^{2}-y_{a, x, 2}^{2} \geqslant\left|y_{a, x}\right|^{2}\left(1-(1-\varepsilon)^{2}\right)$ by (iv). Since $\varepsilon<1$, the conclusion follows from (iii).

(vi) Using (12) and (56), we have 


$$
\begin{aligned}
\left|u\left(x, t_{a, x}\right)\right| & =\left(T-t_{a, x}\right)^{-\frac{1}{p-1}}\left|w_{a}\left(\frac{x-a}{\sqrt{T-t_{a, x}}}, s_{a, x}\right)\right| \\
& =\left(T-t_{a, x}\right)^{-\frac{1}{p-1}}\left|w_{a}\left(Q_{a} y_{a, x}, s_{a, x}\right)\right| .
\end{aligned}
$$

From (i), (v), the monotonicity of $f$ and Proposition 3.1, we have

$$
\begin{aligned}
\left|u\left(x, t_{a, x}\right)\right| & \leqslant\left(T-t_{a, x}\right)^{-\frac{1}{p-1}}\left[f\left(\frac{y_{a, x, 1}}{\sqrt{s_{a, x}}}\right)+C_{0}^{\prime}(1) \frac{\log s_{a, x}}{s_{a, x}}\right] \\
& \leqslant\left(T-t_{a, x}\right)^{-\frac{1}{p-1}}\left[f(\sqrt{\varepsilon})+\varepsilon_{0}\right] \quad \text { (use (v), (i) and (54)) } \\
& =\left(T-t_{a, x}\right)^{-\frac{1}{p-1}}\left[\kappa-\varepsilon_{0}\right] \quad(\text { use (53)). }
\end{aligned}
$$

This concludes the proof of Lemma 4.3 and the proof of (i) of Proposition 4.1.

\section{Step 2: $S$ as the graph of a $C^{\mathbf{1}}$ function}

At the point $a(0)=0$, we know from Proposition 4.1 that $S$ is located along the degenerate direction $Q_{a(0)} e_{2}=e_{2}$. In the following, we will show that $\operatorname{Im} a$ is the graph of $\varphi$, function of the degenerate variable $x_{2}$. Since at each point $b$ of this graph, $S$ is located along the degenerate direction $Q_{b} e_{2}$ which is continuous in terms of $b$, $S \backslash \operatorname{graph} \varphi$ is empty, and $\varphi$ is $C^{1}$. Theorem 1 follows from the following:

Proposition 4.4 ( $S$ as the graph of a $C^{1}$ function). -

(i) There exist $\delta_{1}>0$ and $A \in C\left(\left[-\delta_{1}, \delta_{1}\right], \mathbb{R}^{2}\right)$ such that

$$
\operatorname{Im} A=\left.\operatorname{Im} a\right|_{\left(-\sigma_{0}, \sigma_{0}\right)} \cap \tilde{B}\left(0, \delta_{1}\right)=S \cap \tilde{B}\left(0, \delta_{1}\right),
$$

where $\tilde{B}\left(0, \delta_{1}\right)=\left\{\left(x_{1}, x_{2}\right) \mid\right.$ for $\left.i=1,2,\left|x_{i}\right| \leqslant \delta_{1}\right\}$ and

$$
\forall\left|x_{2}\right| \leqslant \delta_{1}, \quad A\left(x_{2}\right) \cdot e_{2}=x_{2} .
$$

(ii) $A \in C^{1}\left(\left[-\delta_{1}, \delta_{1}\right], \mathbb{R}^{2}\right)$ and the tangent to $S$ at a point $A\left(\bar{x}_{2}\right)$ is $A\left(\bar{x}_{2}\right)+$ $Q_{A\left(\bar{x}_{2}\right)} e_{2} \mathbb{R}$.

Indeed, this implies that locally near $\hat{a}(0)=0, S$ is the graph of a $C^{1}$ function $\varphi$ defined by

$$
\forall\left|x_{2}\right| \leqslant \delta_{1}, \quad A\left(x_{2}\right)=\left(\varphi\left(x_{2}\right), x_{2}\right) .
$$

Therefore, (8) follows from (57), which yields the conclusion of Theorem 1. Let us prove Proposition 4.4.

Proof of Proposition 4.4. -

(i) Consider $\eta_{0}>0$ such that the angle of $\Omega_{0, e_{2}, \eta_{0}}$ is less than $1 / 100$ and than $\alpha_{0}$ where $\alpha_{0}$ is defined in (7). From the uniform cone property of $S$ at points of $\operatorname{Im} a$ (Proposition 4.1), there exists $\delta_{0}>0$ such that $\forall|\sigma|<\sigma_{0}, S \cap B\left(a(\sigma), \delta_{0}\right) \subset \Omega_{a(\sigma), Q_{a(\sigma) e_{2}, \eta_{0}} \text {. }}$

Since $a($.$) and Q_{a(.)} e_{2}$ are continuous, $a(0)=0$ and $Q_{a(0)} e_{2}=e_{2}$ (see (ii) of Proposition 3.1), there exists $\tilde{\sigma}_{0} \leqslant \sigma_{0}$ such that for all $|\sigma|<\tilde{\sigma}_{0}, a(\sigma) \in B\left(0, \frac{\delta_{0}}{2}\right)$, 
$\Omega_{a(\sigma), Q_{a(\sigma)} e_{2}, \eta_{0}} \subset \Omega_{a(\sigma), e_{2}, 2 \eta_{0}}$ and $Q_{a(\sigma)} e_{2} . e_{2} \neq 0$. Hence,

$$
\begin{aligned}
\forall|\sigma|<\tilde{\sigma}_{0}, & a(\sigma) \in B\left(0, \frac{\delta_{0}}{2}\right) \cap \Omega_{0, e_{2}, \eta_{0}}, \\
& S \cap B\left(a(\sigma), \delta_{0}\right) \subset \Omega_{a(\sigma), e_{2}, 2 \eta_{0}} \text { and } \quad Q_{a(\sigma)} e_{2} \cdot e_{2} \neq 0 .
\end{aligned}
$$

Let $\delta_{-}$and $\delta_{+}$be the infimum and the supremum of $\sigma \rightarrow a(\sigma) . e_{2}$ for $|\sigma|<\tilde{\sigma}_{0}$. Since 0 is not an endpoint in $\operatorname{Im} a$ (property (7)), we have

$$
-\frac{\delta_{0}}{2} \leqslant \delta_{-}<0<\delta_{+} \leqslant \frac{\delta_{0}}{2} .
$$

Indeed, $\delta_{-} \geqslant-\frac{\delta_{0}}{2}$ follows from (60). Moreover, if $\delta_{-} \geqslant 0$, then for all $|\sigma|<\tilde{\sigma}_{0}$, $a(\sigma) . e_{2} \geqslant 0$, hence $a\left(-\tilde{\sigma}_{0}, \tilde{\sigma}_{0}\right) \subset \Omega_{0, e_{2}, \eta_{0}} \cap\left\{x_{2} \geqslant 0\right\}$ by (60). This contradicts (7), since the angle of $\Omega_{0, e_{2}, \eta_{0}}$ is less than $\alpha_{0}$. Do the same for $\delta_{+}$.

Now, we are ready to define $A$. If $\delta_{1}=\frac{1}{2} \min \left(\delta_{+},-\delta_{-}\right)>0$, then for all $\left|x_{2}\right| \leqslant \delta_{1}$, there is $\left|\sigma^{*}\left(x_{2}\right)\right| \leqslant \tilde{\sigma}_{0}$ such that $a\left(\sigma^{*}\left(x_{2}\right)\right) \cdot e_{2}=x_{2}$, by continuity of $\sigma \rightarrow a(\sigma) . e_{2}$. If we define $A\left(x_{2}\right)=a\left(\sigma^{*}\left(x_{2}\right)\right)$, then we readily see that (58) holds and if we use (60), then we get

$$
\forall\left|x_{2}\right| \leqslant \delta_{1}, \quad A\left(x_{2}\right)=a\left(\sigma^{*}\left(x_{2}\right)\right) \text { with }\left|\sigma^{*}\left(x_{2}\right)\right| \leqslant \tilde{\sigma}_{0} \quad \text { and } \quad\left|A\left(x_{2}\right)\right|<\frac{\delta_{0}}{2} .
$$

Let us prove that (57) holds. From (62) and (60), we have $\operatorname{Im} A \subset \operatorname{Im} a_{\mid\left(-\tilde{\sigma}_{0}, \tilde{\sigma}_{0}\right)} \subset$ $\Omega_{0, e_{2}, \eta_{0}}$, whose angle is less that $\frac{1}{100}$. Since $A$ is defined for all $\left|x_{2}\right| \leqslant \delta_{1}$, this implies that $\operatorname{Im} A \subset \tilde{B}\left(0, \delta_{1}\right)$. Since $\operatorname{Im} A \subset \operatorname{Im} a_{\mid\left(-\sigma_{0}, \sigma_{0}\right)} \subset S$, (57) holds if we just show that $S \cap \tilde{B}\left(0, \delta_{1}\right) \subset \operatorname{Im} A$, or that for all $b \in S \cap \tilde{B}\left(0, \delta_{1}\right), b=A\left(b . e_{2}\right)$. Remark that for such a $b,\left|b . e_{2}\right| \leqslant \delta_{1}$ so that $A\left(b . e_{2}\right)$ is well defined. Using (62), we write

$$
\left|b-A\left(b . e_{2}\right)\right| \leqslant|b|+\left|A\left(b . e_{2}\right)\right| \leqslant \sqrt{2} \delta_{1}+\frac{\delta_{0}}{2} \leqslant\left(\frac{\sqrt{2}}{4}+\frac{1}{2}\right) \delta_{0}<\delta_{0} .
$$

Therefore, $b \in S \cap B\left(A\left(b . e_{2}\right), \delta_{0}\right) \subset \Omega_{A\left(b . e_{2}\right), e_{2}, 2 \eta_{0}}$ by (62) and (60). Since b.e $e_{2}=$ $A\left(b \cdot e_{2}\right) \cdot e_{2}$ by (58) and the line $x_{2}=A\left(b \cdot e_{2}\right) \cdot e_{2}$ intersects $\Omega_{A\left(b . e_{2}\right), e_{2}, 2 \eta_{0}}$ only at $A\left(b \cdot e_{2}\right)$, this implies that $b=A\left(b . e_{2}\right)$. Thus, (57) holds.

Remains to prove that $A$ is continuous. Consider $\left|x_{2, n}\right| \leqslant \delta_{1}$ such that $x_{2, n} \rightarrow \bar{x}_{2} \in$ $\left[-\delta_{1}, \delta_{1}\right]$ as $n \rightarrow \infty$. Since (62) implies that $A\left(x_{2, n}\right) \in S \cap \bar{B}\left(0, \frac{\delta_{0}}{2}\right)$, a compact set, we may assume that $A\left(x_{2, n}\right) \rightarrow b \in S \cap \bar{B}\left(0, \frac{\delta_{0}}{2}\right)$ (up to a subsequence). Let us show that $b=A\left(\bar{x}_{2}\right)$. Using (62), we have $\left|b-A\left(\bar{x}_{2}\right)\right|<2 \frac{\delta_{0}}{2}$. Therefore, by (62) and (60), we have $b \in \Omega_{A\left(\bar{x}_{2}\right), e_{2}, 2 \eta_{0}}$ on one hand. On the other hand, we have from (58) $A\left(\bar{x}_{2}\right) \cdot e_{2}=\bar{x}_{2}$ and $A\left(x_{2, n}\right) \cdot e_{2}=x_{2, n}$, hence, $b \cdot e_{2}=\bar{x}_{2}=A\left(\bar{x}_{2}\right) \cdot e_{2}$. Since the line $x_{2}=A\left(\bar{x}_{2}\right) \cdot e_{2}$ intersects $\Omega_{A\left(\bar{x}_{2}\right), e_{2}, 2 \eta_{0}}$ only at $A\left(\bar{x}_{2}\right)$, this implies that $b=A\left(x_{2}\right)$ and $A$ is continuous. Thus, (i) of Proposition 4.4 holds.

(ii) Since $A$ is continuous, we learn from the cone property at $A\left(\bar{x}_{2}\right)$ that we can make $\operatorname{Im} A$ (that is the graph of $\varphi$ defined in (59)) as close as we want to the line 
$A\left(\bar{x}_{2}\right)+Q_{A\left(\bar{x}_{2}\right)} e_{2}$ by taking $x_{2}$ close enough to $\bar{x}_{2}$. Therefore, this line is the tangent to the graph of $\varphi$ at $A\left(\bar{x}_{2}\right)$ and $\varphi$ is differentiable at $\bar{x}_{2}$ with

$$
\varphi^{\prime}\left(\bar{x}_{2}\right)=\frac{e_{1} \cdot Q_{A\left(\bar{x}_{2}\right)} e_{2}}{e_{2} \cdot Q_{A\left(\bar{x}_{2}\right)} e_{2}}
$$

(remember that $e_{2} \cdot Q_{A\left(x_{2}\right)} e_{2} \neq 0$ by (62) and (60)). Since $A$ and $b \rightarrow Q_{b}$ are continuous (see (ii) of Proposition 4.1), $\varphi$ is $C^{1}$. This closes the proofs of Proposition 4.4 and Theorem 1.

\subsection{A geometric constraint yielding more regularity for $S$}

We prove Proposition 2 in this subsection.

We first rewrite (iii) of Proposition 3.1 with the terminology of Proposition 4.4. Thanks to (57), $a(\sigma)$ is viewed as $b \in \operatorname{Im} A$. The variable $y_{1}$ is orthogonal to $Q_{b} e_{2}$, the tangent direction to $S$. Therefore, $y_{1}= \pm d\left(y, T_{b}\right)$ where $T_{b}$ is the tangent to $S$ at $b$. More precisely, we have the following:

COROLlaRY 4.5. - For all $K_{0}>0, t \geqslant t_{0} \equiv T-e^{-s_{0}}, b \in \operatorname{Im} A$ and $x \in \mathbb{R}^{2}$ such that $|x-b| \leqslant K_{0} \sqrt{(T-t)|\log (T-t)|}$, we have

$$
\left|(T-t)^{\frac{1}{p-1}} u(x, t)-f\left(\frac{d\left(x, T_{b}\right)}{\sqrt{(T-t)|\log (T-t)|}}\right)\right| \leqslant C_{0}^{\prime}\left(K_{0}\right) \frac{\log |\log (T-t)|}{|\log (T-t)|}
$$

where $T_{b}$ is the tangent to $S$ at $b$.

Proof. - If we introduce $y$ and $s$ such that

$$
s=-\log (T-t) \quad \text { and } \quad y=Q_{b}^{\mathrm{T}}\left(\frac{x-b}{\sqrt{T-t}}\right),
$$

then we see that $|y|=|x-b| / \sqrt{T-t} \leqslant K_{0} \sqrt{s}$ and $s \geqslant s_{0}$. Since $b \in \operatorname{Im} A \subset$ $\left.\operatorname{Im} a\right|_{\left(-\sigma_{0}, \sigma_{0}\right)}$ by (57), we obtain from (iii) of Proposition 3.1

$$
\left|w_{b}\left(Q_{b} y, s\right)-f\left(\frac{y_{1}}{\sqrt{s}}\right)\right| \leqslant C_{0}^{\prime}\left(K_{0}\right) \frac{\log s}{s} .
$$

Remark that we have from (12)

$$
w_{b}\left(Q_{b} y, s\right)=w_{b}\left(\frac{x-b}{\sqrt{T-t}},-\log (T-t)\right)=(T-t)^{\frac{1}{p-1}} u(x, t) .
$$

From (63), we have

$$
\left|y_{1}\right|=\left|y \cdot e_{1}\right|=\left|\frac{x-b}{\sqrt{T-t}} \cdot \tilde{Q}_{b} e_{1}\right| .
$$


Since $Q_{b} e_{1}$ is a normalized normal vector to $S$ (see (ii) of Proposition 4.4), we have $\left|(x-b) \cdot Q_{b} e_{1}\right|=d\left(x, T_{b}\right)$ where $T_{b}$ is the tangent to $S$ at $b$. Therefore,

$$
\left|y_{1}\right|=\frac{d\left(x, T_{b}\right)}{\sqrt{T-t}}
$$

Combining this with (63), (64) and (65) concludes the proof of Corollary 4.5.

Proof of Proposition 2. - Given $x$ near $S$ (= Im A locally), Corollary 4.5 gives different asymptotic behaviors for $u(x, t)$, depending on the choice of the point $b \in$ $\operatorname{Im} A \cap B\left(x, K_{0} \sqrt{(T-t)|\log (T-t)|}\right)$. All these possible behaviors have to agree, up to the error term in Corollary 4.5. This implies a geometric constraint on $S$, which gives some more regularity on $A$ (and $\varphi$ ).

We consider some $\left|x_{2}\right|<\delta_{1}$ and some $h \in \mathbb{R}$ such that $\left|x_{2}+h\right|<\delta_{1}$. Since $A$ is $C^{1}$, there is $C^{*}$ such that

$$
\left|\varphi^{\prime}\left(x_{2}\right)\right| \leqslant C^{*} \quad \text { and } \quad\left|A\left(x_{2}+h\right)-A\left(x_{2}\right)\right| \leqslant C^{*}|h| .
$$

For any time $t>t_{0}$ such that $\left|A\left(x_{2}\right)-A\left(x_{2}+h\right)\right| \leqslant \sqrt{(T-t)|\log (T-t)|}$, we can estimate $u\left(A\left(x_{2}+h\right), t\right)$ from Corollary 4.5 in two ways:

- First by taking $x=b=A\left(x_{2}+h\right)$, which gives

$$
\left|(T-t)^{\frac{1}{p-1}} u\left(A\left(x_{2}+h\right), t\right)-f(0)\right| \leqslant C_{0}^{\prime}(1) \frac{\log |\log (T-t)|}{|\log (T-t)|} .
$$

- Second, by taking $b=A\left(x_{2}\right), x=A\left(x_{2}+h\right)$, which gives

$$
\left|(T-t)^{\frac{1}{p-1}} u\left(A\left(x_{2}+h\right), t\right)-f\left(\frac{d\left(A\left(x_{2}+h\right), T_{A\left(x_{2}\right)}\right)}{\sqrt{(T-t)|\log (T-t)|}}\right)\right| \leqslant C_{0}^{\prime}(1) \frac{\log |\log (T-t)|}{|\log (T-t)|} .
$$

Now, if we fix $t=t\left(x_{2}, h\right)$ such that

$$
\left|A\left(x_{2}+h\right)-A\left(x_{2}\right)\right|=\sqrt{\left(T-t\left(x_{2}, h\right)\right)\left|\log \left(T-t\left(x_{2}, h\right)\right)\right|}
$$

and take $|h|<h_{1}\left(t_{0}\right)$ for some $h_{1}\left(t_{0}\right)>0$, we see from (67) that $t\left(x_{2}, h\right) \geqslant t_{0}$, hence (68) and (69) hold. Therefore,

$$
\left|f(0)-f\left(\frac{d\left(A\left(x_{2}+h\right), T_{A\left(x_{2}\right)}\right)}{\left|A\left(x_{2}+h\right)-A\left(x_{2}\right)\right|}\right)\right| \leqslant 2 C_{0}^{\prime}(1) \frac{\log \left|\log \left(T-t\left(x_{2}, h\right)\right)\right|}{\left|\log \left(T-t\left(x_{2}, h\right)\right)\right|} .
$$

Note that since $A\left(x_{2}\right) \in T_{A\left(x_{2}\right)}$, we have

$$
\frac{d\left(A\left(x_{2}+h\right), T_{A\left(x_{2}\right)}\right)}{\left|A\left(x_{2}+h\right)-A\left(x_{2}\right)\right|} \leqslant 1 .
$$

Therefore, (9) implies that there is $C>0$ such that

$$
C\left[\frac{d\left(A\left(x_{2}+h\right), T_{A\left(x_{2}\right)}\right)}{\left|A\left(x_{2}+h\right)-A\left(x_{2}\right)\right|}\right]^{2} \leqslant\left|f(0)-f\left(\frac{d\left(A\left(x_{2}+h\right), T_{A\left(x_{2}\right)}\right)}{\left|A\left(x_{2}+h\right)-A\left(x_{2}\right)\right|}\right)\right| .
$$


Since $\operatorname{Im} A$ is the graph of $\varphi$, we have

$$
d\left(A\left(x_{2}+h\right), T_{A\left(x_{2}\right)}\right)=\frac{\left|\varphi\left(x_{2}+h\right)-\varphi\left(x_{2}\right)-h \varphi^{\prime}\left(x_{2}\right)\right|}{\sqrt{1+\varphi^{\prime}\left(x_{2}\right)^{2}}} .
$$

If $\tau(d)$ is given by $d=\sqrt{\tau|\log \tau|}$, then

$$
\log \tau \sim 2 \log d \quad \text { and } \quad \log |\log \tau| \sim \log |\log d| \quad \text { as } d \rightarrow 0 .
$$

Therefore,

$$
\frac{\log |\log \tau|}{|\log \tau|} \leqslant \frac{\log |\log d|}{|\log d|}
$$

if $|d| \leqslant d_{0}$ for some $d_{0}>0$. Combining this with (70) and (67), we have for all $\left|x_{2}\right|<\delta_{1}$ and $|h|<h_{0}$ for some $h_{0}>0$ such that $\left|x_{2}+h\right| \leqslant \delta_{1}$,

$$
\frac{\log \left|\log \left(T-t\left(x_{2}, h\right)\right)\right|}{\left|\log \left(T-t\left(x_{2}, h\right)\right)\right|} \leqslant \frac{\log |\log | A\left(x_{2}+h\right)-A\left(x_{2}\right)||}{|\log | A\left(x_{2}+h\right)-A\left(x_{2}\right)||} \leqslant C \frac{\log |\log | h||}{|\log | h||} .
$$

Combining (72), (73), (71), (74) and (67) closes the proof of Proposition 2.

\section{Blow-up profile at a nonisolated blow-up point with the behavior (4)}

We prove Theorem 3 in this section.

\section{Step 1: Asymptotic behavior in self-similar variables around the blow-up set}

We prove (10) in this step. This follows from Corollary 4.5 by taking $b=P_{S}(x)$, the orthogonal projection of $x$ on $S$. Indeed, take $t \geqslant t_{0}$ and $x \in B(0, \delta)$ such that $d(x, S) \leqslant K_{0} \sqrt{(T-t)|\log (T-t)|}$. We define $P_{S}(x)$ as the orthogonal projection of $x$ on $S$. We claim that $P_{S}(x) \in B(0,2 \delta)$, hence $P_{S}(x) \in \operatorname{graph} \varphi=\operatorname{Im} A$ by (8). Indeed, $\left|x-P_{S}(x)\right| \leqslant|x-0|$ since $0 \in S$, therefore, $\left|P_{S}(x)\right| \leqslant\left|P_{S}(x)-x\right|+|x| \leqslant 2|x|<2 \delta$. Since $\left|x-P_{S}(x)\right|=d(x, S) \leqslant K_{0} \sqrt{(T-t)|\log (T-t)|}$ and $d\left(x, T_{P_{S}(x)}\right)=d(x, S),(10)$ follows directly from Corollary 4.5 applied with $b=P_{S}(x)$.

\section{Step 2: Limiting profile in the original set of variables, near the blow-up set}

We prove (11) here. This follows from (10) and the uniform ODE comparison property of Proposition 2.2. Define $t(d)$ such that

$$
d=\sqrt{(T-t(d))|\log (T-t(d))|} .
$$

For all $x \in B(0, \delta) \backslash S$ and $(\xi, \tau) \in \mathbb{R}^{N} \times\left[-\frac{t(d(x, S))}{T-t(d(x, S))}\right.$, 1), we introduce

$$
v(x, \xi, \tau)=(T-\tilde{t})^{\frac{1}{p-1}} u(x+\xi \sqrt{T-\tilde{t}}, \tilde{t}+\tau(T-\tilde{t}))
$$

where $\tilde{t}=t(d(x, S))$. From (10), (76) and Proposition 2.2, we know that there is $\varepsilon_{0}>0$ such that for all $\varepsilon>0$, there is $C_{\varepsilon}$ such that if $d(x, S)<\varepsilon_{0}$ and $(\xi, \tau) \in$ $\mathbb{R}^{N} \times\left[-\frac{t(d(x, S))}{T-t(d(x, S))}, 1\right)$, then 


$$
\begin{aligned}
& \left.\left.\left|\partial_{\tau} v-\right| v\right|^{p-1} v|\leqslant \varepsilon| v\right|^{p}+C_{\varepsilon}(T-t(d(x, S)))^{\frac{p}{p-1}}, \\
& |v(x, 0,0)-f(1)| \leqslant C_{0}^{\prime}(1) \frac{\log |\log (T-t(d(x, S)))|}{|\log (T-t(d(x, S)))|} .
\end{aligned}
$$

Therefore, for all $\varepsilon>0$, there is $\eta(\varepsilon)>0$ such that if $d(x, S)<\eta$, then

$$
\left.\left|\partial_{\tau} v-\right| v\right|^{p-1} v \mid \leqslant \varepsilon\left(|v|^{p}+1\right) \quad \text { and } \quad|v(x, 0,0)-f(1)| \leqslant \varepsilon .
$$

This implies that

$$
\sup _{\tau \in[0,1)}\left|v(x, 0, \tau)-v_{0}(\tau)\right| \rightarrow 0 \quad \text { as } d(x, S) \rightarrow 0
$$

where

$$
v_{0}(\tau)=\left((p-1)(1-\tau)+\frac{(p-1)^{2}}{4 p}\right)^{-\frac{1}{p-1}}
$$

is the solution of $v_{0}^{\prime}(\tau)=v_{0}(\tau)^{p}, v_{0}(0)=f(1)$, defined in particular for all $\tau \in[0,1]$. Moreover,

$$
\sup _{\tau \in[0,1)}\left|\partial_{\tau} v(x, 0, \tau)\right| \leqslant 2 \sup _{\tau \in[0,1]}\left|\partial_{\tau} v_{0}(\tau)\right|
$$

for $d(x, S)$ small. Therefore, for $d(x, S)$ small, $v(x, 0, \tau)$ has a limit as $\tau \rightarrow 1$, hence (76) implies that $u(x, t)$ has a limit $u^{*}(x)$ as $t \rightarrow T$. Using (78) and (76), we see that

$$
u^{*}(x) \sim(T-t(d(x, S)))^{-\frac{1}{p-1}} v_{0}(1) \quad \text { as } d(x, S) \rightarrow 0 .
$$

We claim that

$$
(T-t(d))^{-\frac{1}{p-1}} v_{0}(1) \sim\left(\frac{d^{2}}{|\log d|} \frac{(p-1)^{2}}{8 p}\right)^{-\frac{1}{p-1}} \quad \text { as } d \rightarrow 0
$$

Indeed,

$$
v_{0}(1)=\left(\frac{(p-1)^{2}}{4 p}\right)^{-\frac{1}{p-1}}
$$

from (79), $\log (T-t(d)) \sim 2 \log d$ and $T-t(d) \sim \frac{d^{2}}{2|\log d|}$ as $d \rightarrow 0$ from (75). (11) then follows from (80) and (81). This closes the proof of Theorem 3.

\section{The higher-dimensional case}

We sketch the proof of Theorem 4 here. We need to review the proofs of Theorems 1 , 3 and Proposition 2 to adapt them to the new context. We shall stress the most delicate points in the adaptation of Theorem 1 and Proposition 2. Once this is done, Theorem 3 extends in a natural way to higher dimensions, so we don't discuss it here. 
If $N \geqslant 3$, we consider a nonisolated blow-up point $\hat{a}$ where $u$ has the behavior (4) with $l<N$. We may take $\hat{a}=0$ and $\tilde{Q}_{\hat{a}}=$ Id. According to [24], $S$ satisfies near $\hat{a}$ the $(N-l)$-cone property:

DEFINITION 4.1' (( $N-l)$-cone property and weak tangent plane). - Consider a set $E \subset \mathbb{R}^{N}$.

(i) $E$ is said to have the $(N-l)$-cone property at some point $a \in E$ if there is $\pi a$ $(N-l)$ subspace such that for all $\varepsilon>0$, there is $\delta(a, \varepsilon)>0$ such that

$$
E \cap B(a, \delta) \subset \Omega_{a, \pi, \varepsilon} \equiv\left\{x|| P_{\pi}(x-a)|\geqslant(1-\varepsilon)| x-a \mid\right\},
$$

where $P_{\pi}$ is the orthogonal projection over $\pi . \pi$ is then called the weak tangent plane of $E$ at a.

(ii) $E$ is said to have the uniform $(N-l)$-cone property at some subset $F \subset E$ if for all $\varepsilon>0$ and $a \in F, E$ has the $(N-l)$-cone property at a with $\delta(a, \varepsilon)=\delta(\varepsilon)$.

Remark. $-\Omega_{a, \pi, \varepsilon}$ is a cone with vertex $a$. It shrinks to $a+\pi$ as $\varepsilon \rightarrow 0$.

We have the following consequence of [24]:

LEMMA 6.1 (Velázquez, $\left(N-l_{a}\right)$-cone property for $S$ near a point with the behavior (4)). - If $u$ has the behavior (4) (or (23)) near a, then $S$ satisfies the $(N-l)$-cone property at $a$. The weak tangent plane is spanned by $\tilde{Q}_{a} e_{j}, j=l_{a}+1, \ldots, N$.

Proof. - See Theorem 2 in [24].

Therefore, $S$ has a weak tangent plane at $\hat{a}=0$, spanned by $e_{j}, j=l+1, \ldots, N$. One would expect $S$ to be locally of dimension $N-l$ near $\hat{a}$. However, we are unable to prove that $S$ is a continuum near $\hat{a}$. Therefore, we assume there is $a \in C\left((-1,1)^{N-l}, \mathbb{R}^{N}\right)$ such that $a(0)=\hat{a}=0$ and $\operatorname{Im} a \subset S$ where $\operatorname{Im} a$ is at least $(N-l)$-dimensional in the sense that

$$
\begin{aligned}
& \forall b \in \operatorname{Im} a, \text { there are }(N-l) \text { independent vectors } v_{1}, \ldots, v_{N-l} \text { in } \mathbb{R}^{N} \text { and } \\
& a_{1}, \ldots, a_{N-l} \text { functions in } C^{1}([0,1], S) \text { such that } a_{i}(0)=b \text { and } a_{i}^{\prime}(0)=v_{i} .
\end{aligned}
$$

This hypothesis means that $b$ is actually nonisolated in $(N-l)$ independent directions. We also assume that $\hat{a}=0$ is not an endpoint in $\operatorname{Im} a$ in the sense that

$$
\begin{aligned}
& \forall \varepsilon>0, \text { the projection of } a\left((-\varepsilon, \varepsilon)^{N-l}\right) \text { on the weak tangent plane } \\
& \text { at } \hat{a}=0 \text { contains an open ball with center } \hat{a}=0 .
\end{aligned}
$$

Let us first show the stability result of Section 3 in the case $N \geqslant 3$.

\subsection{Stability of the behavior (4)}

Since we have taken $\hat{a}=0$ and $\tilde{Q}_{\hat{a}}=\mathrm{Id}$, (4) implies that

$$
\sup _{|z| \leqslant K_{0}}\left|(T-t)^{\frac{1}{p-1}} u(z \sqrt{(T-t)|\log (T-t)|}, t)-f\left(\left|z^{\prime}\right|\right)\right| \rightarrow 0 \quad \text { as } t \rightarrow T
$$


where $f$ is defined in (9) and $z=\left(z_{1}, z_{2}, \ldots, z_{N}\right) \equiv\left(z^{\prime}, z_{l+1}, \ldots, z_{N}\right)$. Section 2 then implies that

$$
v_{0} \sim v_{0,2} \quad \text { and } \quad w_{0}(y, s)-\kappa=v_{0}(y, s) \sim \frac{\kappa}{2 p s}\left(l-\frac{\left|y^{\prime}\right|^{2}}{2}\right) \quad \text { as } s \rightarrow \infty,
$$

where $y^{\prime}=\left(y_{1}, \ldots, y_{l}\right)$. We claim that Proposition 3.1 holds here (with the obvious changes $a\left(-\sigma_{0}, \sigma_{0}\right) \rightarrow a\left(\left(-\sigma_{0}, \sigma_{0}\right)^{N-l}\right), 2 \times 2 \rightarrow N \times N$ and $\left.y_{1}^{2} \rightarrow \sum_{i=1}^{l} y_{i}^{2}\right)$.

Proof of Proposition 3.1 in higher dimensions. - Let us follow the 4 steps of the proof given in Section 3.

\section{Step 1: Uniform reduction to a finite-dimensional problem}

This step holds as it is in Section 2.

\section{Step 2: A spectral study of the finite-dimensional problem}

We should define $N$ eigenvalues of $A_{a}(s), l_{a, i}(s), C^{1}$ as functions of $s$. The multifunction $\left\{l_{a, i}(s), i=1, \ldots, N\right\}$ is continuous in terms of $(a, s) \in S \times[-\log T, \infty)$. Corollary 3.1 has to be changed formally. The following corollary crucially uses the $(N-l)$-dimensionality property of $\operatorname{Im} a$.

COROLlaRY 3.1' (Higher-dimensional version of Corollary 3.1). -

(i) (Nonuniform behavior of $v_{a}$ ) For all $|\sigma|<\sigma_{1}$, (23) holds with $l_{a} \leqslant l$. In particular,

$$
A_{a}(s)=-\frac{\beta}{s} \tilde{Q}_{a} I_{l} \tilde{Q}_{a}^{\mathrm{T}}+\mathrm{O}\left(s^{-1-\delta_{a}}\right),
$$

where $I_{l}$ is a $N \times N$ diagonal matrix with $I_{l, i i}=1$ if $i \leqslant l_{a}$ and $I_{l, i i}=0$ if $i \geqslant l_{a}+1$. Moreover, $A_{a}(s)$ has $\left(N-l_{a}\right)$ (hence, at least $(N-l)$ ) eigenvalues equal to $\mathrm{O}\left(s^{-1-\delta_{a}}\right)$, while the others are equal to $-\frac{\beta}{s}+\mathrm{O}\left(s^{-1-\delta_{a}}\right)$.

(ii) (Equations on eigenvalues) For all $\varepsilon>0$, there is $s_{1}(\varepsilon)$ such that for all $|\sigma|<\sigma_{1}$, $i=1, \ldots, N$ and $s \geqslant s_{1}(\varepsilon)$,

$$
\left|l_{a, i}^{\prime}(s)-\frac{1}{\beta} l_{a, i}(s)^{2}\right| \leqslant \varepsilon \sum_{j=1}^{N} l_{a, j}(s)^{2}
$$

where $l_{a, j}(s)$ are the eigenvalues of $A_{a}(s)$.

Proof. - (i) From Proposition 3.1, we have $v_{a} \sim v_{a, 2}$ as $s \rightarrow \infty$ for all $|\sigma|<\sigma_{1}$, hence (23) holds as stated in Section 2.1. Lemma 6.1 then implies that $u$ satisfies the $\left(N-l_{a}\right)$ cone property at $a$ and therefore, $S$ has a $\left(N-l_{a}\right)$-dimensional weak tangent plane at $a$. This plane contains the $(N-l)$ independent directions in property (82). Therefore, $l_{a} \leqslant l$. The estimate for $A_{a}$ then follows from (20), and gives the estimate for eigenvalues.

(ii) See the proof of (ii) of Corollary 3.1.

At the point $a(0)=0$, we know from (84) that $l_{0}=l$. Using Corollary $3.1^{\prime}$, we can rename the eigenvalues of $A_{0}(s)$ such that 


$$
\begin{aligned}
& \forall i=1, \ldots, l, \quad \lambda_{0, i}(s) \sim-\frac{\beta}{s} \text { and } \\
& \forall j=1, \ldots, N-l, \quad \mu_{0, j}(s)=\mathrm{o}\left(\frac{1}{s}\right) \quad \text { as } s \rightarrow \infty .
\end{aligned}
$$

We claim then that Proposition 3.3 holds with eigenvalues $\lambda_{a, i}$ for $i=1, \ldots, l$ and $\mu_{a, j}$ for $j=1, \ldots, N-l$ instead of $\left(\lambda_{a}, \mu_{a}\right)$.

Proof of Proposition 3.3 in higher dimensions. - We should fix a new $\hat{\varepsilon}>0$ and take $s_{3}=s_{1}(\hat{\varepsilon})$ defined in Proposition 3.2. From (85) and the continuity of eigenvalues with respect to $a$, we can find $\sigma_{3} \in\left(0, \sigma_{1}\right)$ where $\sigma_{1}$ appears in Corollary 3.1, such that

$$
\begin{aligned}
\forall i=1, \ldots, l, \quad\left|l_{a, \tau_{a}(i)}\left(s_{3}\right)+\frac{\beta}{s_{3}}\right| \leqslant \frac{\beta}{100 s_{3}}, \\
\forall i=l+1, \ldots, N, \quad\left|l_{a, \tau_{a}(i)}\left(s_{3}\right)\right| \leqslant \frac{\beta}{100 s_{3}},
\end{aligned}
$$

where $\tau_{a}$ is a permutation of $\{1, \ldots, N\}$. If we rename the eigenvalues such that $\lambda_{a, i}(s)=l_{a, \tau_{a}(i)}$ for all $i=1, \ldots, l$ and $\mu_{a, j}=l_{a, \tau_{a}(l+j)}$ for all $j=1, \ldots, N-l$, then we get

$$
\begin{aligned}
& \forall|\sigma| \leqslant \sigma_{3}, \quad \forall i=1, \ldots, l, \quad\left|\lambda_{a, i}\left(s_{3}\right)+\frac{\beta}{s_{3}}\right| \leqslant \frac{\beta}{100 s_{3}}, \\
& \forall j=1, \ldots, N-l, \quad\left|\mu_{a, j}\left(s_{3}\right)\right| \leqslant \frac{\beta}{100 s_{3}} .
\end{aligned}
$$

Lemma 3.2 and its proof hold with the change

$$
N_{a}(s)=\sum_{i=1}^{l} \lambda_{a, i}^{2}+\sum_{j=1}^{N-l} \mu_{a, j}^{2} .
$$

Lemmas 3.3 and 3.4 then hold, with $\left(\lambda_{a}, \mu_{a}\right)$ replaced by $\left(\lambda_{a, i}, \mu_{a, j}\right)$ and different constants. Here comes a delicate point before Lemma 3.5: we need to prove that (31) holds for all $\mu_{a, j}$, for $j=1, \ldots, N-l$. This comes from two arguments. On one hand, we know from Corollary $3.1^{\prime}(\mathrm{i})$ that $A_{a}(s)$ has at least $N-l$ degenerate eigenvalues (that is eigenvalues satisfying (31)). On the other hand, we see from Lemma 3.3 that all degenerate eigenvalues must be in the set of all $\mu_{a, j}, j=1, \ldots, N-l$, which contains $(N-l)$ elements. Therefore, all $\mu_{a, j}$ are degenerate and satisfy (31).

One can easily see that Lemmas 3.5 and 3.6 extend naturally to $N \geqslant 3$ and hold for each $\mu_{a, j}$ and $\lambda_{a, i}$.

\section{Step 3: Solution of the finite-dimensional problem}

Proposition 3.4 holds with " $N \times N$ " instead of " $2 \times 2$ " and $L_{a}=Q_{a} I_{l} Q_{a}^{\mathrm{T}}$ where $I_{l}$ is a $N \times N$ diagonal matrix with $I_{l, i i}=1$ if $i \leqslant l$ and $I_{l, i i}=0$ if $i \geqslant l+1$.

Proof of Proposition 3.4 for $N \geqslant 3$. - If $N \geqslant 3$, one needs to find the asymptotic behavior of all principal minors of order $k$ of the matrix $A_{a}(s)$ with $k=N, \ldots, 1$, before getting the asymptotic behavior of the coefficients of $A_{a}(s)$ (property (46)). 
This has been done by Filippas and Liu [11], Proposition 5.1. The continuity of $b \in$ $a\left(\left(-\sigma_{0}, \sigma_{0}\right)^{N-l}\right) \rightarrow L_{b}$ follows from (46) as in the case $N=2$. Since $L_{a}$ is symmetric, it has $N$ eigenvalues which are the limits as $s \rightarrow \infty$ of $-\frac{s}{\beta} \lambda_{a, i}(i=1, \ldots, l)$ and $-\frac{s}{\beta} \mu_{a, j}(j=1, \ldots, N-l)$, say 1 (multiplicity $l$ ) and 0 (multiplicity $N-l$ ), according to Proposition 3.3. Since $b \rightarrow L_{b}$ is continuous and the multiplicities of eigenvalues 0 and 1 are independent of $b$, we can define continuous eigenvectors for $L_{b}$ (see Oustry and Overton [22], Corollary 2.5). Therefore, we can define a $N \times N$ orthogonal matrix $Q_{b}$, continuous in terms of $b$, diagonaling $L_{b}$. This concludes the proof of Proposition 3.4 in the higher-dimensional case.

\section{Step 4: Asymptotic behavior of $w_{a}$ in $L_{\rho}^{2}$}

This step extends naturally to $N \geqslant 3$, which concludes the proof of Proposition 3.1 .

\subsection{Regularity of the blow-up set}

We prove here the part of Theorem 4 equivalent to Theorem 1. For this, we adapt Section 4 to the case $N \geqslant 3$.

Step 1: The $(N-l)$-cone property for $\operatorname{Im} a$

We claim the following:

Proposition 4.1' (Uniform $(N-l)$-cone property for $S$ at points of $\operatorname{Im} a$ ). -

(i) $S$ has the uniform $(N-l)$-cone property at $a\left(\left(-\sigma_{0}, \sigma_{0}\right)^{N-l}\right)$. The weak tangent plane $\pi_{a(\sigma)}$ at $a(\sigma)$ is spanned by $Q_{a(\sigma)} e_{j}, j=l+1, \ldots, N$, where $e_{j}$ is the $j$ th vector of the canonical basis of $\mathbb{R}^{N}$.

(ii) $Q_{0}=$ Id and the weak tangent plane has an orthogonal basis $\left(Q_{b} e_{j}, j=l+1\right.$, $\ldots, N)$ continuous as a function of $b \in a\left(\left(-\sigma_{0}, \sigma_{0}\right)^{N-l}\right)$.

Remark. - If $j \geqslant l+1$, then $Q_{b} e_{j}$ is a degenerate direction in the asymptotic expansion of (iii) in Proposition 3.1. If $j \leqslant l$, then $Q_{b} e_{j}$ is a nondegenerate direction.

Proof. - (ii) follows directly from (i) by (ii) of Proposition 3.1. The proof of (i) in the case $N=2$ extends naturally to the case $N \geqslant 3$. Just note that $y_{a, x, 1}^{2}$ and $y_{a, x, 2}^{2}$ should be replaced respectively by $\sum_{i=1}^{l} y_{a, x, i}^{2}$ and $\sum_{j=l+1}^{N} y_{a, x, j}^{2}$.

\section{Step 2: $S$ as the graph of a $C^{1}$ function}

The part of Theorem 4 equivalent to Theorem 1 follows from the following:

Proposition $4.4^{\prime}$ ( $S$ as the graph of a $C^{1}$ function). -

(i) There exist $\delta_{1}>0$ and $A \in C\left(\left[-\delta_{1}, \delta_{1}\right]^{N-l}, \mathbb{R}^{N}\right)$ such that

$$
\operatorname{Im} A=a\left(\left(-\sigma_{0}, \sigma_{0}\right)^{N-l}\right) \cap \tilde{B}\left(0, \delta_{1}\right)=S \cap \tilde{B}\left(0, \delta_{1}\right),
$$

where $\tilde{B}\left(0, \delta_{1}\right)=\left\{x|\forall i=1, \ldots, N,| x_{i} \mid \leqslant \delta_{1}\right\}$ and

$$
\begin{aligned}
& \forall\left(x_{l+1}, \ldots, x_{N}\right) \in\left[-\delta_{1}, \delta_{1}\right]^{N-l}, \forall j=l+1, \ldots, N, \\
& A\left(x_{l+1}, \ldots, x_{N}\right) \cdot e_{j}=x_{j} .
\end{aligned}
$$


(ii) $A \in C^{1}\left(\left[-\delta_{1}, \delta_{1}\right]^{N-l}, \mathbb{R}^{N}\right)$ and the tangent plane to $S$ at a point $b \in S \cap \tilde{B}\left(0, \delta_{1}\right)$ is $\pi_{b}$, spanned by $Q_{b} e_{j}, j=l+1, \ldots, N$.

Proof. - The "no-end-point" property of $\hat{a}=0 \operatorname{in} \operatorname{Im} a$ stated in (83) is apparently different from (7) stated for $N=2$. That is why we should carefully define $A$ here. Once this is done, one should follow the case $N=2$ to finish the proof.

From the uniform cone property of $S$ at points of $\operatorname{Im} a$ (Proposition 4.1'), there exists $\delta_{0}>0$ such that $\forall|\sigma|<\sigma_{0}, S \cap B\left(a(\sigma), \delta_{0}\right) \subset \Omega_{a(\sigma), \pi_{a(\sigma)} e_{2}, 1 / 4}$.

Since $a\left(\right.$.) and $Q_{a(.)}$ are continuous, $a(0)=0$ and $Q_{a(0)}=\operatorname{Id}$ (see (ii) of Proposition 3.1), there exists $\tilde{\sigma}_{0} \leqslant \sigma_{0}$ such that for all $|\sigma|<\tilde{\sigma}_{0}, a(\sigma) \in B\left(0, \frac{\delta_{0}}{N}\right), \Omega_{a(\sigma), Q_{a(\sigma)} e_{2}, 1 / 4} \subset$ $\Omega_{a(\sigma), e_{2}, 1 / 3}$ and

$$
\operatorname{det}_{\left(e_{k}, k=l+1, \ldots, N\right)}\left(Q_{a(\sigma)} e_{j}, j=l+1, \ldots, N\right) \neq 0 .
$$

Hence, $\forall|\sigma|<\tilde{\sigma}_{0}$,

$$
\begin{aligned}
& a(\sigma) \in B\left(0, \frac{\delta_{0}}{N}\right) \cap \Omega_{0, e_{2}, 1 / 4}, \quad S \cap B\left(a(\sigma), \delta_{0}\right) \subset \Omega_{a(\sigma), e_{2}, 1 / 3} \quad \text { and } \\
& \operatorname{det}_{\left(e_{k}, k=l+1, \ldots, N\right)}\left(Q_{a(\sigma)} e_{j}, j=l+1, \ldots, N\right) \neq 0 .
\end{aligned}
$$

Since $Q_{a(0)}=\mathrm{Id}$, we learn from Proposition 4.1' that the weak tangent plane at $a(0)=0$ is $\pi_{0}$, spanned by $e_{j}, j=l+1, \ldots, N$. Using property (83), we find $\delta_{1}\left(\tilde{\sigma}_{0}\right)>0$ such that $\tilde{B}_{\pi_{0}}\left(0, \delta_{1}\right) \subset P_{\pi_{0}}\left(a\left[-\tilde{\sigma}_{0}, \tilde{\sigma}_{0}\right]^{N-l}\right)$, where

$$
\tilde{B}_{\pi_{0}}\left(0, \delta_{1}\right)=\left\{x \in \pi_{0}|| x_{j} \mid \leqslant \delta_{1}, \text { for } j=l+1, \ldots, N\right\} .
$$

This implies that $A$ can be defined on $\tilde{B}_{\pi_{0}}\left(0, \delta_{1}\right)$, so that (87) holds. One can then finish the proof of Proposition 4.4' by just following the proof of Proposition 4.4.

\subsection{A geometric constraint yielding some more regularity}

This subsection is dedicated to the higher-dimensional version of Proposition 2. Note that from Proposition 4.4', $\operatorname{Im} A$ is the graph of $\varphi \in C^{1}\left(\left[-\delta_{1}, \delta_{1}\right]^{N-l}, \mathbb{R}^{l}\right)$ such that

$$
\forall\left(x_{l+1}, \ldots, x_{N}\right) \in\left[-\delta_{1}, \delta_{1}\right]^{N-l}, \quad A\left(x_{l+1}, \ldots, x_{N}\right)=\left(\varphi_{1}, \ldots, \varphi_{l}, x_{l+1}, \ldots, x_{N}\right),
$$

where $\varphi_{i}=\varphi_{i}\left(x_{l+1}, \ldots, x_{N}\right)$. Proposition 2 holds with $\varphi\left(x_{2}\right)$ replaced by $\varphi_{i}\left(x_{l+1}, \ldots, x_{N}\right)$ and $\varphi^{\prime}$ by $\nabla \varphi_{i}$.

Proof of Proposition 2 in higher dimensions. - Just follow the case $N=2$ with $A\left(x_{2}\right)$ replaced by $A(\tilde{x})$ where $\tilde{x}=\left(x_{l+1}, \ldots, x_{N}\right)$. The only delicate point is the equivalent of (73) which is: for all $i=1, \ldots, l$,

$$
d\left(A(\tilde{x}+\tilde{h}), \pi_{A(\tilde{x})}\right) \geqslant \frac{\left|\varphi_{i}(\tilde{x}+\tilde{h})-\varphi_{i}(\tilde{x})-\tilde{h} \cdot \nabla \varphi_{i}(\tilde{x})\right|}{\sqrt{1+\left|\nabla \varphi_{i}(\tilde{x})\right|^{2}}} .
$$

Note that we just need this inequality in our argument. 
To prove (89), just note that $\operatorname{Im} A \subset S_{i}$, the surface of equation $x_{i}=\varphi_{i}\left(x_{l+1}, \ldots, x_{N}\right)$. Hence, $\pi_{A(\tilde{x})} \subset \pi_{i, A(\tilde{x})}$, the $(N-1)$-dimensional tangent plane to $S_{i}$ at $A(\tilde{x})$. Therefore, $d\left(A(\tilde{x}+\tilde{h}), \pi_{A(\tilde{x})}\right) \geqslant d\left(A(\tilde{x}+\tilde{h}), \pi_{i, A(\tilde{x})}\right)$, equal to the right-hand side of (89) (standard calculations).

\section{Appendix}

\section{A. Reduction of the asymptotic blow-up behavior to a finite-dimensional problem}

We prove Proposition 3.2 here. The use of the Liouville theorem is crucial here. The proof is the same as in the proof of Propositions 1.11 and 1.12 in [8]. All is about stability, with respect to initial data in [8], and with respect to the blow-up point here. For this reason, we just sketch the proof and refer to [8] for the proofs.

We know from (25) that $w_{0}(y, s) \rightarrow \kappa$ as $s \rightarrow \infty$ in $L_{\rho}^{2}$. If $a$ is a blow-up point near 0 , we know from (15) that $w_{a} \rightarrow \pm \kappa$ as $s \rightarrow \infty$. Only $+\kappa$ is selected because of the following local constant sign property for $u$, which is a direct consequence of the positivity of $u$ for $x=0$, and the ODE comparison of Proposition 2.2.

Lemma A.1 (Constant sign property of $u$ for $(x, t)$ close to $(0, T)$ ). - There exists $\delta>0$ such that

$$
\forall t \in[T-\delta, T), \forall x \in B(0, \delta), \quad u(x, t) \geqslant 0 .
$$

Proof. - See Corollary 1.8 in [8].

The Liouville theorem of Proposition 2.1 allows us to show that $v_{a} \equiv w_{a}-\kappa \rightarrow 0$ as $s \rightarrow \infty$ in $L_{\rho}^{2}$, uniformly for $a$ in a neighborhood of 0 . This uniformity is a central argument in our proof.

LEMMA A.2 (Uniform smallness of $v_{a}$ ). - There exists $\tilde{\sigma}_{2}>0$ such that

(i) $\sup _{|\sigma|<\tilde{\sigma}_{2}}\left\|v_{a}(s)\right\|_{L_{\rho}^{2}} \rightarrow 0$ as $s \rightarrow \infty$,

(ii) for all $R>0$, $\sup _{|\sigma|<\tilde{\sigma}_{2}}$ ( $\left.\sup _{|y|<R}\left|v_{a}(y, s)\right|\right) \rightarrow 0$ as $s \rightarrow \infty$.

Proof. - See Proposition 1.10 in [8].

Note that this lemma yields the first estimate of Proposition 3.2. We know from (22) that for each $a$, either $v_{a} \sim v_{a, 2}$ or $v_{a} \sim v_{a,-}$ as $s \rightarrow \infty$. (25) shows that $v_{0} \sim v_{0,2}$, its neutral mode, as $s \rightarrow \infty$. The predominance of the neutral mode turns out to be a stable behavior for solutions of (17). Indeed:

LEMMA A.3 (Uniform stability of the dynamics where $v_{2}$ is predominant). - There exists $\tilde{\sigma}_{4}$ with the following property:

(i) There exists $s^{*}$ such that for all $|\sigma|<\tilde{\sigma}_{4}$ and $s_{0} \geqslant s^{*}$,

$$
\text { if } X_{a}\left(s_{0}\right) \geqslant Y_{a}\left(s_{0}\right)+Z_{a}\left(s_{0}\right), \text { then } \forall s \geqslant s_{0}, X_{a}(s) \geqslant \frac{1}{2}\left(Y_{a}(s)+Z_{a}(s)\right)
$$

where

$$
\begin{aligned}
& X_{a}(s)=\left\|v_{a, 2}(s)\right\|_{L_{\rho}^{2}}, \quad Y_{a}(s)=\left\|v_{a,-}(s)\right\|_{L_{\rho}^{2}}+\left\||y|^{k / 2} v_{a}\right\|_{\rho}, \\
& Z_{a}(s)=\left\|v_{a,+}(s)\right\|_{L_{\rho}^{2}}
\end{aligned}
$$


and $k=k(M)>0$ (where $M$ is defined in (14)) is fixed.

(ii) For all $\varepsilon>0$, there exists $s_{3}(\varepsilon)$ such that for all $|\sigma|<\tilde{\sigma}_{4}$ and $s \geqslant s_{3}(\varepsilon)$,

$$
Y_{a}(s)+Z_{a}(s) \leqslant \varepsilon X_{a}(s) .
$$

Proof. - See Proposition 1.11 in [8].

This lemma yields the second estimate of Proposition 3.2, namely the fact that $v_{a} \sim v_{a, 2}$ as $s \rightarrow \infty$, uniformly in $a(\sigma)$. Therefore, the study of (17) reduces to the study of its projection over the neutral mode, $v_{a, 2}$, where, of course, one should take into account the quadratic term. This leads to the equation stated in Proposition $3.2\left(A_{a}\right.$ is the matrix of the components of $v_{a, 2}$, thanks to (21)). See Proposition 1.12 in [8] for a proof.

This closes the proof of Proposition 3.2.

\section{B. A refined equation on the neutral mode of (17)}

We prove Lemma 3.4 here. We first prove (29). We claim the following:

ClAim B.1 (Control of $v_{a}(s)$ in $L_{\rho}^{r}$ ). - For all $r>1$, there exists $C(r)>0$ and $s_{0}^{*}(r)>0$ such that for all $|\sigma|<\sigma_{3}$ and $s \geqslant s_{3}+s_{0}^{*}(r)$,

$$
\left(\int\left|v_{a}(y, s)\right|^{r} \rho(y) \mathrm{d} y\right)^{1 / r} \leqslant \frac{C(r)}{s} .
$$

Proof. - If $r=2$, we use Lemma 3.3 to get $\left|A_{a}(s)\right| \leqslant C s^{-1}$, hence $\left\|v_{a, 2}(s)\right\|_{L_{\rho}^{2}} \leqslant C s^{-1}$ by (21). Since $\left\|v_{a}(s)\right\|_{L_{\rho}^{2}} \sim\left\|v_{a, 2}(s)\right\|_{L_{\rho}^{2}}$ as $s \rightarrow \infty$ (uniformly for $|\sigma|<\sigma_{3}$ ), we get the estimate with some $s_{0}^{*}(2)$.

If $r \neq 2$, we combine the case $r=2$ with the following regularizing effect of the operator $\mathcal{L}$.

Claim B.2 (Herrero-Velázquez). - If $v_{a}$ satisfies (17), then for all $r>1$, there exists $\tilde{s}_{0}(r)$ and $C(r, M)$ such that

$$
\left(\int\left|v_{a}(y, s)\right|^{r} \rho(y) \mathrm{d} y\right)^{1 / r} \leqslant C\left(\int\left|v_{a}\left(y, s-\tilde{s}_{0}\right)\right|^{2} \rho(y) \mathrm{d} y\right)^{1 / 2} .
$$

Proof. - See Lemma 2.3 in [14]. This closes the proof of Claim B.1.

Eq. (29) follows if we prove that for all $|\sigma|<\sigma_{3}$ and $s \geqslant s_{3}^{\prime}$ for some $s_{3}^{\prime} \geqslant s_{3}$,

$$
\begin{aligned}
& z_{a}^{\prime}(s) \geqslant \frac{1}{2} z_{a}(s)-\frac{C}{s^{2}}, \\
& y_{a}^{\prime}(s) \leqslant-\frac{1}{2} y_{a}(s)+\frac{C}{s^{2}},
\end{aligned}
$$

where $z_{a}(s)=\left\|v_{a,+}(s)\right\|_{L_{\rho}^{2}}$ and $y_{a}(s)=\left\|v_{a,-}(s)\right\|_{L_{\rho}^{2}}$. Indeed, integrating (90) between $s$ and $+\infty$ and (91) between $s_{3}^{\prime}$ and $s$, we obtain for all $|\sigma|<\sigma_{3}$ and $s \geqslant s_{3}^{\prime}$, 


$$
\begin{aligned}
& z_{a}(s) \leqslant C e^{s / 2} \int_{s}^{\infty} e^{-t / 2} t^{-2} \mathrm{~d} t \leqslant C^{\prime} s^{-2}, \\
& y_{a}(s) \leqslant e^{-\left(s-s_{3}^{\prime}\right) / 2} y_{a}\left(s_{3}^{\prime}\right)+C \int_{s_{3}^{\prime}}^{s} e^{-(s-t) / 2} t^{-2} \mathrm{~d} t<C^{\prime} s^{-2} .
\end{aligned}
$$

(Note that $y_{a}(s)+z_{a}(s) \leqslant\left\|v_{a}(s)\right\|_{L_{\rho}^{2}} \leqslant\left\|v_{a}(s)\right\|_{L^{\infty}} \leqslant M+\kappa$ by (14).) Now we prove (90). (91) follows in the same way.

Projecting (17) on the positive eigenspace of $\mathcal{L}$, we get

$$
\partial_{s} v_{a,+}=\mathcal{L} v_{a,+}+P_{+}\left(f\left(v_{a}\right)\right),
$$

where $P_{+}$is the corresponding $L_{\rho}^{2}$ orthogonal projector. Multiplying this by $v_{a,+} \rho$ and integrating over $\mathbb{R}^{N}$, we get

$$
\begin{aligned}
\frac{1}{2} \frac{\mathrm{d}}{\mathrm{d} s} \int v_{a,+}^{2} \rho & =\int \mathcal{L} v_{a,+} \cdot v_{a,+} \rho+\int P_{+}\left(f\left(v_{a}\right)\right) v_{a,+} \rho \\
& \geqslant \frac{1}{2} \int v_{a,+}^{2} \rho-\left(\int v_{a,+}^{2} \rho\right)^{1 / 2}\left(\int P_{+}\left(f\left(v_{a}\right)\right)^{2} \rho\right)^{1 / 2},
\end{aligned}
$$

where we used the Cauchy-Schwartz inequality.

Since $P_{+}$is a $L_{\rho}^{2}$ projector and $f$ is quadratic, we have

$$
\left(\int\left[P_{+}\left(f\left(v_{a}\right)\right)\right]^{2} \rho\right)^{1 / 2} \leqslant\left(\int\left(f\left(v_{a}\right)\right)^{2} \rho\right)^{1 / 2} \leqslant C\left(\int\left(v_{a}\right)^{4} \rho\right)^{1 / 2} .
$$

Using Claim B.1, we have for all $|\sigma|<\sigma_{3}$ and $s \geqslant s_{3}+s_{0}^{*}(4),\left(\int v_{a}^{4} \rho\right)^{1 / 2} \leqslant C(4) s^{-2}$. Thus, (90) follows with $s_{3}^{\prime}=s_{3}+s_{0}^{*}(4)$. This closes the proof of (29).

Now we prove (30). The estimate on eigenvalues is obtained by evaluating the estimate on $A_{a}$ at eigenfunctions. Therefore, we just focus on $A_{a}$. With (29) and Claim B.1, we are ready to get a refined equation on $A_{a}(s)$.

Using (20) and (17), we write for all $|\sigma|<\sigma_{3}$ and $s \geqslant s_{3}$,

$$
A_{a}^{\prime}(s)=E_{1}+E_{2}+E_{3}
$$

where

$$
\begin{aligned}
& E_{1}=\frac{p}{2 \kappa} \int v_{a, 2}(y, s)^{2} M(y) \rho(y) \mathrm{d} y, \\
& E_{2}=\frac{p}{2 \kappa} \int\left(v_{a}^{2}-v_{a, 2}^{2}\right) M(y) \rho(y) \mathrm{d} y, \\
& E_{3}=\int g\left(v_{a}\right) M(y) \rho(y) \mathrm{d} y .
\end{aligned}
$$

By straightforward calculations, we get from (21)

$$
E_{1}=\frac{1}{\beta} A_{a}(s)^{2} \quad \text { where } \beta=\frac{\kappa}{2 p} .
$$


Recalling that $v=v_{a,+}+v_{a, 2}+v_{a,-}$ (see (19)), we write

$$
\begin{aligned}
\left|E_{2}\right| & \leqslant C \int\left|v_{a}^{2}-v_{a, 2}^{2}\right||M(y)| \rho(y) \mathrm{d} y \\
& =C \int\left|v_{a,+}+v_{a,-}\right|\left|v_{a}+v_{a, 2}\right||M(y)| \rho(y) \mathrm{d} y \\
& \leqslant C\left(\int\left|v_{a,+}+v_{a,-}\right|^{2} \rho\right)^{1 / 2}\left(\int\left|v_{a}+v_{a, 2}\right|^{4} \rho\right)^{1 / 4}\left(\int|M(y)|^{4} \rho\right)^{1 / 4}
\end{aligned}
$$

where we used Hölder's inequality.

Using (29), Claim B.1, and the norm equivalence in the finite-dimensional space (18) where $v_{a, 2}$ lays $\left(\int\left|v_{a, 2}\right|^{4} \rho \leqslant C\left(\int\left|v_{a, 2}\right|^{2} \rho\right)^{2} \leqslant C\left(\int\left|v_{a}\right|^{2} \rho\right)^{2}\right.$ by Proposition 3.1), we endup with

$$
\left|E_{2}\right| \leqslant C s^{-3} \text { for all } s \geqslant s_{3}^{\prime \prime}
$$

for some $s_{3}^{\prime \prime}>s_{3}$.

We finally estimate $E_{3}$. From (20), (17) and the Cauchy-Schwartz inequality, we write

$$
\begin{aligned}
\left|E_{3}\right| & \leqslant \int\left|g\left(v_{a}\right)\right||M(y)| \rho(y) \mathrm{d} y \\
& \leqslant C\left(\int\left|v_{a}\right|^{6} \rho\right)^{1 / 2}\left(\int|M(y)|^{2} \rho(y) \mathrm{d} y\right)=C\left(\int\left|v_{a}\right|^{6} \rho\right)^{1 / 2} .
\end{aligned}
$$

Using Claim B.1, we end-up with

$$
\left|E_{3}\right| \leqslant C s^{-3} \quad \text { for all } s \geqslant s_{3}+s_{0}^{*}(6) .
$$

Combining (92), (93), (94) and (95), we obtain the first inequality in (30), for all $|\sigma|<\sigma_{3}$ and $s \geqslant \tilde{s}_{3}$ for some $\tilde{s}_{3} \geqslant s_{3}$. This closes the proof of Lemma 3.4.

\section{Asymptotic behavior in the $\frac{y}{\sqrt{s}}$ variable}

We prove (iii) of Proposition 3.1 here. We follow Velázquez's work in [24] (Proposition 2.3), with a special care to the convergence speed and to the uniformity with respect to blow-up points.

Define

$$
\varphi(y, s)=\left(p-1+\frac{(p-1)^{2}}{4 p} \frac{y_{1}^{2}}{s}\right)^{-\frac{1}{p-1}}+\frac{\kappa}{2 p s} \quad \text { and } \quad q_{a}=w_{a}-\varphi .
$$

Straightforward calculations based on (13) and (i) of Proposition 3.1 yield for all $|\sigma|<\sigma_{0}$ and $s \geqslant s_{0}$,

$$
\forall y \in \mathbb{R}^{N}, \quad \partial_{s} q_{a}=(\mathcal{L}+V) q_{a}+B\left(q_{a}\right)+R(y, s),\left\|q_{a}(s)\right\|_{L_{\rho}^{2}} \leqslant C_{0} \frac{\log s}{s^{2}},
$$

where 


$$
\begin{aligned}
\mathcal{L} & =\Delta-\frac{1}{2} y \cdot \nabla+1, \\
V(y, s) & =p \varphi^{p-1}-\frac{p}{p-1}, \\
B(q) & =|\varphi+q|^{p-1}(\varphi+q)-\varphi^{p}-p \varphi^{p-1} q, \\
R(y, s) & =-\partial_{s} \varphi+\Delta \varphi-\frac{1}{2} y \cdot \nabla \varphi-\frac{\varphi}{p-1}+\varphi^{p} .
\end{aligned}
$$

Let us introduce the following norm

$$
L_{\rho}^{2, r}(\psi)=\sup _{|\xi|<r}\left(\int|\psi(y)|^{2} \rho(y-\xi) \mathrm{d} y\right)^{1 / 2} .
$$

Velázquez's idea in [24] is to make estimates on solutions of (96) in the $L_{\rho}^{2, r(\tau)}$ norm where $r(\tau)=K_{0} e^{\left(\tau-s_{0}\right) / 2} \leqslant K_{0} \sqrt{\tau}$. The following is proved in [24]:

LEMmA C.1. - For all $C_{3}>0$ and $K_{3}>0$, there is $s_{3}\left(C_{3}, K_{3}\right)>0$ such that if $Z(y, s)$ satisfies

$$
\begin{aligned}
& \partial_{s} Z \leqslant\left(\mathcal{L}+\frac{C_{3}}{s}\right) Z+C_{3}\left(Z^{2}+\frac{1+|y|^{2}}{s^{2}}+1_{\left\{|y|>2 K_{3} \sqrt{s}\right\}}\right), \\
& 0 \leqslant Z(y, s) \leqslant C_{3},
\end{aligned}
$$

for all $(y, s) \in \mathbb{R}^{N} \times\left[s_{3}, \infty\right)$, then, for all $s^{\prime} \geqslant s_{3}$ and $\tau \in\left[s^{\prime}, s\right]$, we have

$$
g(\tau) \leqslant C_{4}\left(C_{3}\right)\left[e^{\tau-s^{\prime}}\left(\left\|Z\left(s^{\prime}\right)\right\|_{L_{\rho}^{2}}+\frac{\log s^{\prime}}{s^{\prime 2}}\right)+\int_{s^{\prime}}^{\left(\tau-2 K_{3}\right)_{+}} \frac{e^{\tau-t-2 K_{3}} g(t)^{2}}{\left(1-e^{-\left(\tau-t-2 K_{3}\right)}\right)^{1 / 20}} \mathrm{~d} t\right],
$$

where $g(\tau)=L_{\rho}^{2, r\left(K_{3}, \tau, s^{\prime}\right)}(Z(\tau)), r\left(K_{3}, \tau, s^{\prime}\right)=K_{3} e^{\left(\tau-s^{\prime}\right) / 2}, e^{\left(s-s^{\prime}\right) / 2}=\sqrt{s}$ and $h_{+}=$ $\max (h, 0)$.

Proof. - See Proposition 2.3 in [24] (in particular, pp. 1575-1581).

Let us apply this to our case (96). It is readily seen from (97) that for all $K_{0}>0$, there is $C_{2}\left(K_{0}, M\right)$ ( $M$ is defined in (14)) such that

$$
\begin{aligned}
& V(y, s) \leqslant \frac{C_{2}}{s}, \quad|B(q)| \leqslant C_{2}\left(|q|^{2}+1_{\left\{|y| \geqslant 2 K_{0} \sqrt{s}\right\}}\right), \\
& |R(y, s)| \leqslant C_{2}\left(\frac{1+|y|^{2}}{s^{2}}+1_{\left\{|y| \geqslant 2 K_{0} \sqrt{s}\right\}}\right)
\end{aligned}
$$

(one may consider first the case $|y| \geqslant 2 K_{0} \sqrt{s}$ and then $|y| \leqslant 2 K_{0} \sqrt{s}$ and make a Taylor expansion for $\xi=y / \sqrt{s}$ bounded).

If $Z_{a}=\left|q_{a}\right|$, then we use Kato's inequality $\Delta g \cdot \operatorname{sgn}(g) \leqslant \Delta(|g|)$ to derive from (96) and (99) the following: 
For all $K_{0}$, there is $C_{2}\left(K_{0}, M\right)>0$ such that for all $|\sigma|<\sigma_{0}$ and $s \geqslant s_{0}$,

$$
\begin{aligned}
\forall y \in \mathbb{R}^{N}, \quad \partial_{s} Z_{a} \leqslant\left(\mathcal{L}+\frac{C_{2}}{s}\right) Z_{a}+C_{2}\left(Z_{a}^{2}+\frac{1+|y|^{2}}{s^{2}}+1_{\left\{|y| \geqslant 2 K_{0} \sqrt{s}\right\}}\right), \\
\left\|Z_{a}(s)\right\|_{L_{\rho}^{2}} \leqslant C_{0} \frac{\log s}{s^{2}} .
\end{aligned}
$$

We claim the following:

CLAIM C.2. - For all $K_{0}>0$, there exists $C_{6}>0$ and $s_{6}$ such that for all $|\sigma|<\sigma_{0}$ and $s \geqslant s_{6}$,

$$
L_{\rho}^{2, K_{0} \sqrt{s}}\left(Z_{a}(s)\right) \leqslant C_{6} \frac{\log s}{s} .
$$

Proof. - Consider $K_{0}>0$. Fix $s_{5}\left(K_{0}\right) \geqslant 2 \max \left(s_{3}\left(C_{2}\left(K_{0}, M\right), K_{0}\right), s_{0}\right)$ such that for all $s \geqslant s_{5}, \frac{s}{2}-\log s \geqslant 0$ where $C_{2}, s_{3}$ and $M$ are defined in (100), (14) and Lemma C.1. If $s \geqslant s_{5}$ and $s^{\prime}$ is defined by $e^{\left(s-s^{\prime}\right) / 2}=\sqrt{s}$, then it is readily seen that $s^{\prime}=s-\log s \geqslant$ $\frac{s}{2} \geqslant \max \left(s_{3}, s_{0}\right)$. Therefore, we have from (96), (100) and Lemma C.1: for all $|\sigma|<\sigma_{0}$, for all $\tau \in\left[s^{\prime}, s\right]$,

$$
g(\tau) \leqslant C_{5}^{\prime}\left(C_{0}, K_{0}, M\right)\left[e^{\tau-s^{\prime}} \frac{\log s^{\prime}}{s^{\prime 2}}+\int_{s^{\prime}}^{\left(\tau-2 K_{0}\right)_{+}} \frac{e^{\tau-t-2 K_{0}} g(t)^{2}}{\left(1-e^{-\left(\tau-t-2 K_{0}\right)}\right)^{1 / 20}} \mathrm{~d} t\right]
$$

where $g(\tau)=L_{\rho}^{2, r\left(K_{0}, \tau, s^{\prime}\right)}\left(Z_{a}(\tau)\right)$ and $r\left(K_{0}, \tau, s^{\prime}\right)=K_{0} e^{\left(\tau-s^{\prime}\right) / 2}$.

By a standard Gronwall estimate (see Lemma 2.2 in [24]), there is $s_{6}\left(C_{0}, K_{0}\right) \geqslant s_{5}$ and $C_{5}\left(C_{0}, K_{0}\right)>C_{5}^{\prime}$ such that if $s \geqslant s_{6}$, then for all $\tau \in\left[s^{\prime}, s\right], g(\tau) \leqslant C_{5} e^{\tau-s^{\prime}} \frac{\log s^{\prime}}{s^{\prime 2}}$.

If $\tau=s$, then we get $L_{\rho}^{2, K_{0} \sqrt{s}}\left(Z_{a}(s)\right) \leqslant C_{5} e^{s-s^{\prime}} \frac{\log s^{\prime}}{s^{\prime 2}} \leqslant 2 C_{5} \frac{\log s}{s}$. This finishes the proof of Claim C.2.

To conclude the proof, we use the following result from [24]:

Claim C.3. - Assume that $Z$ satisfies (98), then

$$
\sup _{|y| \leqslant \frac{K_{3}}{2} \sqrt{s}}\left|Z_{a}(y, s)\right| \leqslant C\left(C_{3}, K_{3}\right) L_{\rho}^{2, K_{3} \sqrt{s}}\left(Z_{a}(s)\right) .
$$

Proof. - See Proposition 2.3 in [24] (in particular, p. 1581).

Thus, for all $|\sigma|<\sigma_{0}$ and $s \geqslant s_{6}$,

$$
\sup _{|y| \leqslant \frac{K_{0}}{2} \sqrt{s}}\left|Z_{a}(y, s)\right| \leqslant C\left(K_{0}, C_{0}, M\right) \frac{\log s}{s} .
$$

Since

$$
\left|w_{a}(y, s)-f_{1}\left(\frac{y}{\sqrt{s}}\right)\right| \leqslant Z_{a}+\frac{\kappa}{2 p s},
$$

this concludes the proof of (iii) of Proposition 3.1. 


\section{Acknowledgements}

The author wants to thank T. Colding, R.V. Kohn, F.H. Lin and F. Merle for interesting conversations and remarks about the paper. He wants to acknowledge partial support he received from the NSF grant DMS-9631832.

\section{REFERENCES}

[1] J.M. Ball, Remarks on blow-up and nonexistence theorems for nonlinear evolution equations, Quart. J. Math. Oxford (Ser. 2) 28 (112) (1977) 473-486.

[2] A.J. Bernoff, A.L. Bertozzi, T.P. Witelski, Axisymmetric surface diffusion: dynamics and stability of self-similar pinchoff, J. Statist. Phys. 93 (3-4) (1998) 725-776.

[3] M.D. Betterton, M.P. Brenner, Collapsing bacterial cylinders, Preprint.

[4] M.P. Brenner, P. Constantin, L.P. Kadanoff, A. Schenkel, S.C. Venkataramani, Diffusion, attraction and collapse, Nonlinearity 12 (4) (1999) 1071-1098.

[5] J. Bricmont, A. Kupiainen, Universality in blow-up for nonlinear heat equations, Nonlinearity 7 (2) (1994) 539-575.

[6] S.J. Chapman, B.J. Hunton, J.R. Ockendon, Vortices and boundaries, Quart. Appl. Math. 56 (3) (1998) 507-519.

[7] K. Deng, H.A. Levine, The role of critical exponents in blow-up theorems: the sequel, J. Math. Anal. Appl. (2000).

[8] C. Fermanian Kammerer, F. Merle, H. Zaag, Stability of the blow-up profile of non-linear heat equations from the dynamical system point of view, Math. Annalen 317 (2) (2000) 195-237.

[9] C. Fermanian Kammerer, H. Zaag, Boundedness up to blow-up of the difference between two solutions to a semilinear heat equation, Nonlinearity 13 (4) (2000) 1189-1216.

[10] S. Filippas, R.V. Kohn, Refined asymptotics for the blowup of $u_{t}-\Delta u=u^{p}$, Comm. Pure Appl. Math. 45 (7) (1992) 821-869.

[11] S. Filippas, W.X. Liu, On the blowup of multidimensional semilinear heat equations, Ann. Inst. H. Poincaré Anal. Non Linéaire 10 (3) (1993) 313-344.

[12] H. Fujita, On the blowing up of solutions of the Cauchy problem for $u_{t}=\Delta u+u^{1+\alpha}$, J. Fac. Sci. Univ. Tokyo Sect. I 13 (1966) 109-124.

[13] Y. Giga, R.V. Kohn, Nondegeneracy of blowup for semilinear heat equations, Comm. Pure Appl. Math. 42 (6) (1989) 845-884.

[14] M.A. Herrero, J.J.L. Velázquez, Blow-up behaviour of one-dimensional semilinear parabolic equations, Ann. Inst. H. Poincaré Anal. Non Linéaire 10 (2) (1993) 131-189.

[15] T. Kato, Perturbation Theory for Linear Operators, Springer, Berlin, 1995. Reprint of the 1980 edition.

[16] H.A. Levine, Some nonexistence and instability theorems for solutions of formally parabolic equations of the form $P u_{t}=-A u+F(u)$, Arch. Rational Mech. Anal. 51 (1973) 371-386.

[17] F. Merle, Solution of a nonlinear heat equation with arbitrarily given blow-up points, Comm. Pure Appl. Math. 45 (3) (1992) 263-300.

[18] F. Merle, H. Zaag, Reconnection of vortex with the boundary and finite time quenching, Nonlinearity 10 (6) (1997) 1497-1550.

[19] F. Merle, H. Zaag, Stability of the blow-up profile for equations of the type $u_{t}=\Delta u+$ $|u|^{p-1} u$, Duke Math. J. 86 (1) (1997) 143-195.

[20] F. Merle, H. Zaag, Optimal estimates for blowup rate and behavior for nonlinear heat equations, Comm. Pure Appl. Math. 51 (2) (1998) 139-196. 
[21] F. Merle, H. Zaag, A Liouville theorem for vector-valued nonlinear heat equations and applications, Math. Annalen 316 (1) (2000) 103-137.

[22] F. Oustry, M.L. Overton, Variational analysis of the total projection for symmetric matrices, 2000.

[23] H.M. Soner, P.E. Souganidis, Singularities and uniqueness of cylindrically symmetric surfaces moving by mean curvature, Comm. Partial Differential Equations 18 (5-6) (1993) 859-894.

[24] J.J.L. Velázquez, Higher-dimensional blow up for semilinear parabolic equations, Comm. Partial Differential Equations 17 (9-10) (1992) 1567-1596.

[25] J.J.L. Velázquez, Classification of singularities for blowing up solutions in higher dimensions, Trans. Amer. Math. Soc. 338 (1) (1993) 441-464.

[26] J.J.L. Velázquez, Estimates on the $(n-1)$-dimensional Hausdorff measure of the blow-up set for a semilinear heat equation, Indiana Univ. Math. J. 42 (2) (1993) 445-476.

[27] H. Zaag, One-dimensional behavior of singular $N$-dimensional solutions of semilinear heat equations, Preprint, 2001. 